\title{
Approximating the Set of Pareto Optimal Solutions in Both the Decision and Objective Spaces by an Estimation of Distribution Algorithm
}

\author{
Aimin Zhou, Qingfu Zhang and Yaochu Jin
}

\begin{abstract}
Most existing multiobjective evolutionary algorithms aim at approximating the Pareto front (PF), the distribution of the Pareto optimal solutions in the objective space. In many reallife applications, however, a good approximation to the Pareto set (PS), the distribution of the Pareto optimal solutions in the decision space, is also required by a decision maker. This paper considers a class of multiobjective optimization problems (MOPs), in which the dimensionalities of the PS and the PF manifolds are different so that a good approximation to the PF might not approximate the PS very well. It proposes a probabilistic model based multiobjective evolutionary algorithm, called MMEA, for approximating the PS and the PF simultaneously for an MOP in this class. In the modeling phase of MMEA, the population is clustered into a number of subpopulations based on their distribution in the objective space, the PCA technique is used to estimate the dimensionality of the PS manifold in each subpopulation, and then a probabilistic model is built for modeling the distribution of the Pareto optimal solutions in the decision space. Such a modeling procedure could promote the population diversity in both the decision and objective spaces. MMEA is compared with three other methods, KP1, OmniOptimizer and RM-MEDA on a set of test instances, five of which are proposed in this paper. The experimental results clearly suggest that overall, MMEA performs significantly better than the three compared algorithms in approximating both the PS and the PF.
\end{abstract}

Index Terms-Multiobjective optimization, Pareto optimality, estimation of distribution algorithm, principal component analysis.

\section{INTRODUCTION}

This paper considers the following continuous multiobjective optimization problem (continuous MOP):

$$
\begin{array}{cc}
\operatorname{minimize} & F(x)=\left(f_{1}(x), \ldots, f_{m}(x)\right)^{T} \\
\text { subject to } & x \in \prod_{i=1}^{n}\left[a_{i}, b_{i}\right]
\end{array}
$$

where $-\infty<a_{i}<b_{i}<+\infty$ for all $i=1, \ldots, n$. $\prod_{i=1}^{n}\left[a_{i}, b_{i}\right] \subset$ $R^{n}$ is the decision space and $x=\left(x_{1}, \ldots, x_{n}\right)^{i=1} \in R^{n}$ is the decision variable vector. $F: \prod_{i=1}^{n}\left[a_{i}, b_{i}\right] \rightarrow R^{m}$ consists of $m$ real-valued continuous objective functions $f_{i}(x), i=$ $1, \ldots, m . R^{m}$ is the objective space.

A. Zhou and Q. Zhang are with the School of Computer Science \& Electronic Engineering, University of Essex, Wivenhoe Park, Colchester, CO4 3SQ, U.K. (e-mail:\{azhou,qzhang\}@essex.ac.uk).

Y. Jin is with Honda Research Institute Europe, Carl-Legien-Str. 30, 63073 Offenbach, Germany. (e-mail:yaochu.jin@honda-ri.de).
Let $u=\left(u_{1}, \ldots, u_{m}\right)^{T}, v=\left(v_{1}, \ldots, v_{m}\right)^{T} \in R^{m}$ be two vectors, $u$ is said to dominate $v$, if $u \neq v$ and $u_{i} \leq v_{i}$ for all $i=1, \ldots, m . x^{\star}$ is called (globally) Pareto optimal if there is no other $x$ such that $F(x)$ dominates $F\left(x^{\star}\right)$. The set of all the Pareto optimal points, denoted by PS, is called the Pareto set. The image of the PS on the objective space, $\mathrm{PF}=\left\{y \in R^{m} \mid y=F(x), x \in \mathrm{PS}\right\}$, is called the Pareto front [1], [2].

Most existing multiobjective evolutionary algorithms (MOEAs) aim at approximating PFs [2]-[16]. However, in some real-world applications, particularly when the preference (i.e. utility function) of a decision maker is not clearly defined, a good approximation to both the PF and the PS should be required by the decision maker for facilitating their decision making as argued in [17]-[20]. For example, if two objectives $f_{1}$ and $f_{2}$ are much more important than objective $v$ in engineering design, one often needs to first optimize $f_{1}$ and $f_{2}$ and obtain a good approximation to both the PF and the PS, then finds from the approximate PS a solution that optimizes $v$ subject to certain constraints as their final solution. In some cases, a good approximation to the PF might not approximate the PS well. Two typical classes of continuous MOPs, in which the approximation of their PSs should be carefully addressed, are as follows:

- Class I: A finite number of different points in the PS may have the same image in the PF under the mapping $F$ from the PS to the PF, but the PS and the PF are of the same dimensionality. ZDT6 [21], Jin1 [22] and the SYM-PART instances [23] are test instances in this class. In all these instances, the PS consists of a number of disconnected continuous $(m-1)$-D manifolds.

- Class II: The PF is an $(m-1)-\mathrm{D}$ continuous manifold and the PS is a continuous manifold of a higher dimensionality. All the inverse images of a point in the PF could constitute a non-zero dimensional continuous manifold. Some WFG test instances [24] belong to this class. For example, in WFG6, the PF is a 1-D continuous curve in the objective space, while the PS is a 2-D rectangle. The inverse image of a point in the $\mathrm{PF}$ is a 1-D curve in the decision space.

To generate a good approximation to both the PS and the PF of an MOP, an MOEA should arguably have an effective mechanism to encourage and maintain the population diversity, not only in the objective space as most MOEAs do, but also in the decision space. For this reason, Deb and Tiwari 
[25] introduced the crowding distance in the decision space into the nondominated sorting scheme in Omni-Optimizer, a generalization of NSGA-II [26], for promoting the population diversity in the decision space. Chan and Ray [27] suggested using two selection operators in MOEAs, one encourages the diversity in the objective space and the other does so in the decision space. They implemented KP1 and KP2, two algorithms using these two selection operators. It should be pointed out that the MOPs that KP1, KP2 and Omni-Optimizer attempt to deal with are of Class I. Preuss et al [28] and Rudolph et al [23] also proposed to use a restart strategy for finding a good approximation to the PS of an MOP of Class I. To the best of our knowledge, no effort has been made for dealing with problems of Class II. The major purpose of this paper is to study how to approximate both the PS and the PF of an MOP of Class II.

In [14], we studied a "regular" continuous MOP in which both the PF and the FS are piecewise $(m-1)$-D continuous manifolds, and proposed RM-MEDA, an estimation of distribution algorithm (EDA) for approximating its PF. In this paper, we generalize the idea of RM-MEDA and propose a probabilistic model based multiobjective evolutionary algorithm, called MMEA, for approximating the PS and the PF of an MOP of Class II simultaneously. MMEA has the following features:

- The population diversity in the decision space is promoted in its reproduction generator, instead of in the selection operators as in Omni-Optimizer, KP1 and KP2. The NDSselection, which is used in RM-MEDA, is employed in MMEA.

- To build a probabilistic model of promising solutions, the population is divided, based on their distribution in the objective space, into a number of subpopulations. Therefore, the population diversity in the objective space can be promoted. To ease the burden of tuning the number of subpopulations, a random strategy is used for setting it.

- The principal component analysis (PCA) technique is used to estimate the dimensionality of the PS manifold in each subpopulation, and then a probabilistic model can be built for modeling the distribution of promising solutions in the decision space. In such a way, the population diversity in the decision space can be encouraged.

The rest of the paper is organized as follows: Section II gives the details of the algorithm. Section III presents the performance metrics and the test instances, some of which are proposed in this paper for the first time. Section IV compares MMEA with KP1, Omni-Optimizer and RM-MEDA on these test instances. More discussions on the ability of MMEA are provided in Section V. Section VI concludes this paper and suggests some future research topics.

\section{Algorithm}

\section{A. Framework}

At each generation, the proposed algorithm, MMEA, maintains:
- A population of $N$ solutions (i.e. points in $\prod_{i=1}^{n}\left[a_{i}, b_{i}\right]$ ):

$$
x^{1}, \ldots, x^{N} .
$$

- Their function values: $F\left(x^{1}\right), \ldots, F\left(x^{N}\right)$.

MMEA adopts the following widely used EDA framework:

Phase 1 Initialization: Generate an initial population $P$ and compute the $F$-values of these solutions in $P$.

Phase 2 Modeling: Build a model for modeling the distribution of the individuals in $P$.

Phase 3 Reproduction: Generate a set of new solutions $Q$ by sampling from the model built in Phase 2 and compute the function values of these solutions in $Q$.

Phase 4 Selection: Select $N$ solutions from $P \cup Q$ and replace all the solutions in $P$ by them.

Phase 5 Stopping Condition: If a stopping condition is met, stop and return all the solutions in $P$ and their corresponding $F$-values. Otherwise, go to Phase 2.

In the following, we give and discuss the details of modeling, reproduction and selection.

\section{B. Modeling}

In a successful algorithm for approximating both the PS and the PF of (1), the individuals in its population should approximate the PS in the decision space and their images should converge to the PF in the objective space as the search goes on. Therefore, one could model the PS and the PF based on information extracted from the population. Such models can be further used for sampling new good solutions. This idea has been used to some extent in RM-MEDA. The problem that RM-MEDA was designed for is a "regular" continuous MOP, in which both the PS and the PF are of the same dimensionality. In this paper, the same idea is used in the modeling phase of MMEA for dealing with an MOP of Class II.

The modeling phase in MMEA works as follows:

Step 1 Building a Utopian PF: Based on information from the current population $P$, build an $(m-1)$-D simplex in the objective space as a Utopian PF.

Step 2 Determining the Number of Subpopulations: Determine $K$, the number of subpopulations used in modeling the PS.

Step 3 Selecting Reference Points: Set $Y^{1}, \ldots, Y^{K}, K$ points which are uniformly spread on the Utopian $\mathrm{PF}$ in the objective space, to be $K$ reference points.

Step 4 Clustering: Cluster the population $P$ into $K$ subpopulations $P^{1}, \ldots, P^{K}$.

Step 5 Principal Component Analysis and Modeling: Perform PCA on each subpopulation $P^{k}, k=$ $1, \ldots, K$ and build a model for it.

In the following, we give the details of the major steps in the above modeling phase.

1) Building a Utopian PF: We assume that the PF of the MOP in question is of $(m-1)$-D. Therefore, it is reasonable to use an $(m-1)$-D simplex as a Utopian PF. The following procedure is used to construct such a simplex $S$ : 


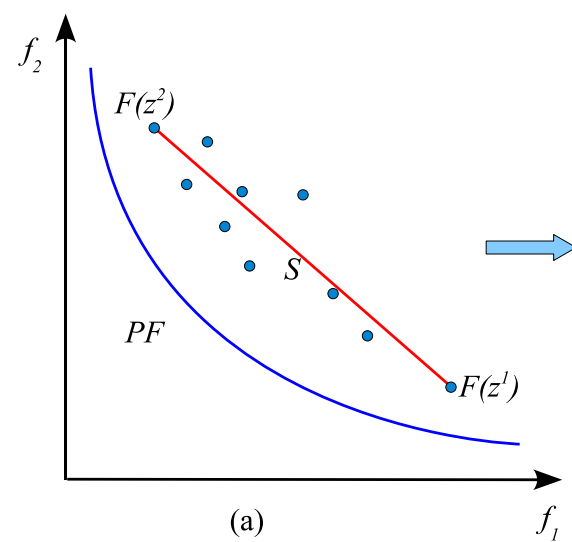

(a)

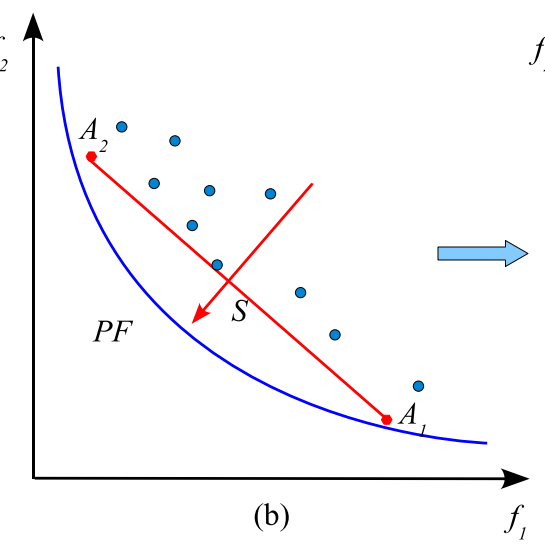

(b)

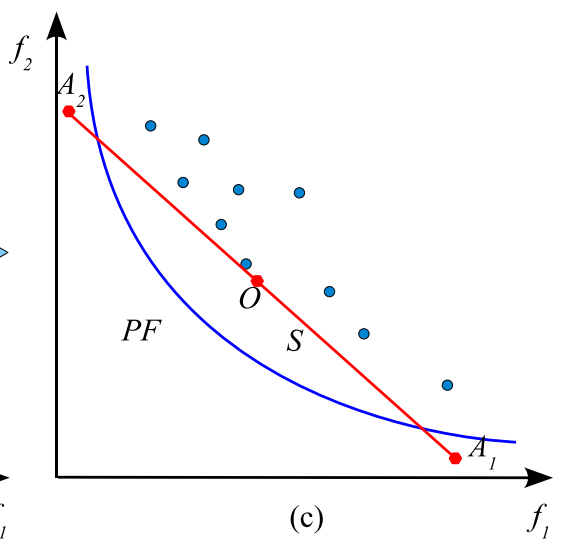

Fig. 1. Illustration of building a Utopian PF in the case of two objectives. (a) Find the extreme points and initialize simplex $S$. (b) Move $S$ along its normal direction. (c) Enlarge $S$.

Step 1.1 For $i=1, \ldots, m$, find the individual solution $z^{i}$ in $P$ such that $z^{i}$ is a nondominated solution in $P$ and it has the largest $f_{i}$ function value among all the nondominated solutions in $P$.

Step 1.2 Initialize $S$ as the $(m-1)$-D simplex with vertexes $F\left(z^{1}\right), \ldots, F\left(z^{m}\right)$ in the objective space. Move $S$ along its normal direction to a position such that (a) no point in $S$ can be dominated by any solutions in $P$, and (b) the moved distance should be as short as possible.

Step 1.3 Let $A_{1}, \ldots, A_{m}$ be the vertexes of the moved simplex $S$. Compute the center of $S$

$$
O=\frac{1}{m} \sum_{i=1}^{m} A_{i} .
$$

Then enlarge $S$ by moving its vertexes

$$
A_{i}:=A_{i}+\left[(1+\alpha)^{\frac{1}{m-1}}-1\right]\left(A_{i}-O\right)
$$

for $i=1, \ldots, m$.

$\alpha>0$ is a control parameter. It is easy to work out that the volume of the simplex $S$ is increased by $100 \alpha \%$ in Step 1.3. The major reason why we enlarge $S$ is to guide the algorithm to extend its search in the objective space. When $m$, the number of the objectives is 2, $S$ is a 1-D line segment in the objective space. Fig. 1 illustrates how $S$ is generated in this case.

2) Determining the Number of Subpopulations: To reduce the problem-dependence of $K$, the value of $K$ is uniformly randomly chosen from the set $\left\{1,2, \ldots, K_{\max }\right\} . K_{\max }$ is a control parameter.

3) Selecting Reference Points: It is desirable that reference points uniformly spread on the Utopian PF. Note that the Utopian PF is a simplex, in our implementation, we use the simplex point picking method [29] for selecting reference points.

4) Clustering: For each reference point $Y^{k}$ obtained in Step 3, we select a number of points from $P$ closer to it for forming $P^{k}$ as follows:
Step 4.1 Compute, in the objective space, the Euclidian distances between $Y^{k}$ to all the individual solutions in $P$.

Step 4.2 Select the $\min \left\{N,\left[\frac{2 N}{K}\right]\right\}$ closest solutions to $Y^{k}$ and let them constitute subpopulation $P^{k}$.

In clustering, different subpopulations may overlap, which could improve the search performance in between different reference points.

5) PCA and Modeling: The individual solutions in subpopulation $P^{k}, k=1, \ldots, K$ should, hopefully, scatter around the PS in the decision space as the search goes on. For simplicity, we can model the subpopulation $P^{k}$ as a hyper-cuboid $\Phi^{k}$ in the decision space and regard each individual in $P^{k}$ as an observation of the following random vector:

$$
\xi=\zeta+\varepsilon,
$$

where $\zeta$ is uniformly randomly distributed on $\Phi^{k}, \varepsilon \sim$ $N\left(0, \sigma^{k} I\right)$ is an $n$-dimensional zero-mean Gaussian vector, $I$ is the $n \times n$ identity matrix and $\sigma^{k}>0$.

Now the task is how to estimate $\Phi^{k}$ and $\sigma^{k}$. We do it as follows:

Step 5.1 Compute the sample mean and the sample covariance matrix of the individual solutions in $P^{k}$ :

$$
\bar{x}^{k}=\frac{1}{\left|P^{k}\right|} \sum_{x \in P^{k}} x
$$

and

$$
\operatorname{Cov}^{k}=\frac{1}{\left|P^{k}\right|-1} \sum_{x \in P^{k}}\left(x-\bar{x}^{k}\right)\left(x-\bar{x}^{k}\right)^{T}
$$

where $\left|P^{k}\right|$ is the cardinality of $P^{k}$.

Step 5.2 Compute the eigenvalues of $\operatorname{Cov}^{k}$ :

$$
\lambda_{1}^{k} \geq \lambda_{2}^{k} \geq \ldots \geq \lambda_{n}^{k}
$$

and their corresponding unity eigenvectors:

$$
V_{1}^{k}, V_{2}^{k}, \ldots, V_{n}^{k}
$$


Step 5.3 Set $n^{k}$, the dimensionality of the hyper-cuboid $\Phi^{k}$ to be the smallest integer such that

$$
\sum_{j=1}^{n^{k}} \lambda_{j}^{k} \geq \theta \sum_{j=1}^{n} \lambda_{j}^{k}
$$

where the threshold $0 \leq \theta \leq 1$ is an algorithm parameter.

Step 5.4Compute the range of the projections of the points in $P^{k}$ onto the first $n^{k}$ principal component directions:

$$
l_{j}^{k}=\min _{x \in P^{k}}\left\{\left(x-\bar{x}^{k}\right)^{T} V_{j}^{k}\right\}
$$

and

$$
u_{j}^{k}=\max _{x \in P^{k}}\left\{\left(x-\bar{x}^{k}\right)^{T} V_{j}^{k}\right\}
$$

for $j=1, \ldots, n^{k}$.

Step 5.5 Set

$$
\begin{gathered}
\Phi^{k}=\left\{x \in R^{n} \mid x=\bar{x}^{k}+\sum_{j=1}^{n^{k}} c_{j} V_{j}^{k},\right. \\
l_{j}^{k}-\frac{(1+\beta)^{\frac{1}{n^{k}}}-1}{2}\left(u_{j}^{k}-l_{j}^{k}\right) \leq c_{j} \\
\leq u_{j}^{k}+\frac{(1+\beta)^{\frac{1}{n^{k}}}-1}{2}\left(u_{j}^{k}-l_{j}^{k}\right), \\
\left.j=1, \ldots, n^{k}\right\}
\end{gathered}
$$

where $\beta$ is a control parameter.

Step 5.6 Set

$$
\sigma^{k}=\frac{1}{n-n^{k}} \sum_{j=n^{k}+1}^{n} \lambda_{j}^{k} .
$$

The dimensionality of the PS is unknown, neither is that of $\Phi^{k}$. In Step 5.3, the dimensionality of $\Phi^{k}$ is set such that $\Phi^{k}$ holds at least $100 \theta \%$ of the variation in the solutions in $P^{k}$. In Step 5.5, $\Phi^{k}$ is enlarged along each of the first $n^{k}$ principal component directions such that its volume is $100 \beta \%$ larger than that the smallest $n^{k}$-D hyper-cuboid containing the projections of all the solutions of $P^{k}$ on the space spanned by $V_{1}^{k}, \ldots, V_{n^{k}}^{k}$ from $\bar{x}^{k}$. The motivation behind this extension is to extrapolate the points in $P^{k}$ for searching unexplored promising areas in the decision space. $\varepsilon$ is modeled as a Gaussian noise vector and all its components are i.i.d., which facilitates the sampling procedure.

The reference points in the objective space used in clustering are hopefully uniformly distributed along the PF, therefore they could guide the search to generate a good approximation to the PF in the objective space. The modeling in the decision space attempts to model the PS, which enables the algorithm to generate a set of points for approximating the PS in the decision space.

The three major differences in the modeling phase between RM-MEDA and MMEA are:

- RM-MEDA uses the local PCA [30] technique to partition the population into several clusters. In contrast, MMEA in this paper selects the subpopulation centers from the Utopian PF and performs clustering based on the distances in the objective space, which is computationally cheaper. Moreover, the local PCA could not be applied in MMEA since the dimensionality of the PS manifold must be predetermined in the local PCA and it is unknown in the problems MMEA aims to solve.

- The number of clusters is preset in RM-MEDA, while MMEA in this paper chooses the number of subpopulations randomly, which lightens the burden of tuning this control parameter.

- In modeling each subpopulation, RM-MEDA sets the dimensionality of the PS manifold to be $(m-1)$, while MMEA needs to estimate it. This difference is due to the fact that these two algorithms are for different MOPs.

\section{Sampling}

A new solution $x$ is generated in Phase 4 of MMEA as follows:

Step 1 Uniformly randomly generate an integer $k$ from $\{1,2, \ldots, K\}$.

Step 2 Uniformly randomly generate a point $x^{\prime}$ from $\Phi^{k}$. Generate a noise vector $\varepsilon^{\prime}$ from $N\left(0, \sigma^{k} I\right)$.

Step 3 Set $y=x^{\prime}+\varepsilon^{\prime}$, and let the new solution $x$ as

$$
x_{j}=\left\{\begin{array}{ll}
y_{j} & \text { if } a_{j} \leq y_{j} \leq b_{j} \\
\frac{1}{2}\left(z_{j}+a_{j}\right) & \text { if } y_{j}<a_{j} \\
\frac{1}{2}\left(z_{j}+b_{j}\right) & \text { if } y_{j}>b_{j}
\end{array},\right.
$$

where $j=1, \cdots, n$, and $z$ is a randomly selected solution from the subpopulation $P^{k}$.

In our implementation, the above procedure is repeated $N$ times for generating $N$ solutions in Phase 4.

\section{Selection}

The selection operator used in the experimental studies is the NDS-selection, a variant of non-dominated sorting scheme [26] proposed in [14]. It works as follows:

Step 1 Set $Q=P \cup Q$ and $P=\emptyset$.

Step 2 Partition $Q$ into different fronts $F_{1}, \ldots, F_{l}$ by using the fast non-dominated sorting approach [26]. Set $k=0$.

Do

$$
\begin{aligned}
& k=k+1, \\
& P=P \cup F_{k},
\end{aligned}
$$$$
\text { Until }|P| \geq N \text {. }
$$

Step 3 While $|P|>N$, Do

For all the individual members in $F_{k} \cap P$, compute their crowding distances in $F_{k} \cap P$. Remove the element in $F_{k} \cap P$ with the smallest crowding distance from $P$. In the case when there is more than one member with the smallest crowding distance, randomly choose one and remove it.

In Step 2, the NDS-selection partitions $Q$ into different fronts $F_{1}, \ldots, F_{l}$ such that the $j$-th front $F_{j}$ contains all the non-dominated solutions in $\{P \cup Q\} \backslash\left(\cup_{i=1}^{j-1} F_{i}\right)$. Therefore, there is no solution in $\{P \cup Q\} \backslash\left(\cup_{i=1}^{j-1} F_{i}\right)$ that could dominate a solution in $F_{j}$.

The crowding distance, used in Step 3, of point $x$ is defined as the average side length of the largest $m$-D rectangle in the 
objective space subject to two constraints: (a) each of its sides is parallel to a coordinate axis, and (b) $F(x)$ is the only interior point of the rectangle among all the points in $\left\{F(y) \mid y \in F_{k} \cap\right.$ $P\}$. A solution with a larger crowding distance is given priority to be selected since it could increase the population diversity in the objective space.

\section{Test Instances And Performance Metrics}

\section{A. Test Instances}

MMEA is for approximating both the PS and the PF of an MOP of Class II. WFG instances are the only Class II instances that we have found in the literature. Two WFG instances: WFG6 and WFG7, have been used in our experiments. Based on the experiments in [24], the PFs of WFG7 could be "easily" and "quickly" found by NSGA-II, while WFG6 is "hard" for NSGA-II. It can be because the objectives in WFG7 are separable while it is not the case in WFG6. The PSs of these two test instances are a 2-D rectangle in the decision space when their control parameters are set as in Table I. To study the behaviors of MOEAs on nonlinear PSs, we have designed several new MOP test instances of Class II with nonlinear PSs. All these test instances are listed in Table I. Figs. 2 and 3 plot their PFs and the projections of their PSs onto lower-dimensional spaces.

\section{B. Performance Metrics}

The Inverted Generational Distance (IGD) metric [14], [31] and hypervolume difference $\left(I_{H}^{-}\right)$metric [32] are used to assess the algorithm performances in our experimental studies.

Let $P^{*}$ be a set of uniformly distributed Pareto optimal points in the PF (or PS). Let $P$ be an approximation to the $\mathrm{PF}$ (or the PS). The IGD metric is defined as follows,

$$
\operatorname{IGD}\left(P^{*}, P\right)=\frac{\sum_{v \in P^{*}} d(v, P)}{\left|P^{*}\right|}
$$

where $d(v, P)$ is a distance between $v$ and $P$ and $\left|P^{*}\right|$ is the cardinality of $P^{*}$.

We denote $I G D$ metric as $I G D F$ when $P^{*}$ is a set of points in the $\mathrm{PF}$ and $d(v, P)$ is the Euclidian distance in the objective space, and as $I G D X$ when $P^{*}$ is a set of points in the PS and $d(v, P)$ is the Euclidian distance in the decision space.

The $I_{H}^{-}$metric is defined as

$$
I_{H}^{-}\left(P^{*}, P\right)=I_{H}\left(P^{*}\right)-I_{H}(P)
$$

where $I_{H}(P)$ is the hypervolume between the set $P$ and a bounded reference point [33].

Both the $I G D$ metric and the $I_{H}^{-}$metric measure convergence and diversity. To have low $I G D$ and $I_{H}^{-}$values, $P$ must be close to the PF (or PS) and cannot miss any part of the whole PF (or PS).

In our experiments, 1,000 points, in which $f_{1}$ or $t$ taking 1,000 equidistant values from their lower bounds to their upper bounds, are selected from the respective PFs of F1-F6 to be $P^{\star}$ for computing the IGDF metrics. $50 \times 50=2,500$ points in the PF of F7 with $s, t=\frac{0}{49} \times \frac{\pi}{2}, \frac{1}{49} \times \frac{\pi}{2}, \ldots, \frac{49}{49} \times \frac{\pi}{2}$, are taken to form $P^{\star}$ for computing the $I G D F$ metric for experiments on F7. $50 \times 50=2,500$ points in the respective PSs of F1-F5, in which $x_{1}$ and $x_{2}$ take 50 equidistant values from their lower bounds to their upper bounds respectively, are taken to form $P^{\star}$ for computing the $I G D X$ metrics. $25 \times 25 \times 25=15,625$ points in the respective PSs of F6 and F7, in which $x_{1}, x_{2}$ and $x_{3}$ take 25 equidistant values from their lower bounds to their upper bounds respectively, are taken to be $P^{\star}$ for computing the $I G D X$ metrics. In calculating the $I_{H}^{-}$values, the bounded reference point is chosen to be $(2.4,4.5)^{T}$ for $\mathrm{F} 1$ and $\mathrm{F} 2$, $(1.2,1.2)^{T}$ for F3-F6 and $(1.2,1.2,1.2)^{T}$ for F7.

\section{EXPERIMENTAL RESULTS}

\section{A. Experimental Settings and Algorithms in Comparison}

TABLE II

EXPERIMENTAL SETTINGS FOR F1-F7

\begin{tabular}{lll}
\hline \hline & number of variables & $n=20$ \\
& population size for each algorithm & $N=250$ (F1-F5) \\
& & $N=500$ (F6-F7) \\
& number of generations & 500 \\
& number of runs for each algorithm & 20 \\
& & \\
& crossover parameter in SBX & $\eta_{c}=20$ \\
Omni & crossover rate & $P_{c}=0.8$ \\
KP1 & parameter in polynomial mutation & $\eta_{m}=20$ \\
& mutation rate & $P_{m}=1 / n$ \\
RM-MEDA & & $K=10$ \\
& number of clusters & \\
& $\alpha$ in building a Utopian PF (Step 1.3) & $\alpha=1.0$ \\
MMEA & $\beta$ in PCA and modeling (Step 5.5) & $\beta=1.0$ \\
& $\theta$ in PCA and modeling (Step 5.3) & $\theta=0.8$ \\
& $K_{\max \text { in selecting reference points }}$ & $K_{\max }=30$ \\
\hline \hline
\end{tabular}

The studies in [23], [27], [28] have shown that popular MOEAs, such as PAES [34], NSGA-II [26] and SPEA2 [35], cannot approximate both the PF and the PS simultaneously since these methods could not maintain the population diversity in the decision space. MOEA/D, a recent MOEA based on aggregation proposed in [14], can not do so either for the same reason. In our experiments, we compare MMEA with KP1 [27] ${ }^{1}$ and Omni-Optimizer [25] ${ }^{2}$. As mentioned in Introduction, both KP1 and Omni-Optimizer try to approximate both the PF and the PS of an MOP by promoting the population diversity in the decision space in their selection operators. The simulated binary crossover (SBX) [36] and the polynomial mutation [37] are used in these two methods for generating offspring. Since MMEA is based on RMMEDA [14], we also compare MMEA with RM-MEDA on these problems.

Table II lists all the parameter settings in our experiments. The population in each algorithm is initialized uniformly and randomly in the decision space. All the following results are based on 20 independent runs of each algorithm on each test instance.

\footnotetext{
${ }^{1}$ We use KP1 in this paper because the experimental results in [27] have shown that KP1 is slightly better than KP2.

${ }^{2}$ The $\mathrm{C}++$ source codes of KP1 was obtained from its authors and OmniOptimizer was implemented by ourselves.
} 
TABLE I

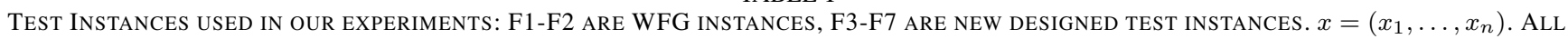
THESE INSTANCES BELONG TO CLASS II.

\begin{tabular}{|c|c|c|c|}
\hline Instance & Range of $x_{i}$ & Objectives, PS and PF & Remarks \\
\hline$F 1$ & {$[0,2 i]$} & $\begin{array}{l}W F G 6(M=2, k=2)[24] \\
\text { PS: } x_{i}=0.7 i, \text { for } i=3, \ldots, n, 0 \leq x_{1} \leq 2,0 \leq x_{2} \leq 4 \\
\text { PF: } f_{1}=2 \sin (t), f_{2}=4 \cos (t), 0 \leq t \leq 0.5 \pi\end{array}$ & $\begin{array}{l}\text { PS is a 2-D rectangle. } \\
\mathrm{PF} \text { is concave. } \\
\text { two objectives. }\end{array}$ \\
\hline$F 2$ & {$[0,2 i]$} & $\begin{array}{l}W F G 7(M=2, k=2)[24] \\
\text { PS: } x_{i}=0.7 i, \text { for } i=3, \ldots, n, 0 \leq x_{1} \leq 2,0 \leq x_{2} \leq 4 \\
\text { PF: } f_{1}=2 \sin (t), f_{2}=4 \cos (t), 0 \leq t \leq 0.5 \pi\end{array}$ & $\begin{array}{l}\text { PS is a 2-D rectangle. } \\
\text { PF is concave. } \\
\text { two objectives. }\end{array}$ \\
\hline$F 3$ & {$[0,1]$} & $\begin{array}{l}f_{1}(x)=\left(x_{1}+x_{2}\right) / 2, \\
f_{2}(x)=g(x)\left(1-\sqrt{\frac{f_{1}}{g}}\right), \\
\text { where } g(x)=1+\frac{5}{n-2} \sum_{i=3}^{n} h\left(x_{i}\right)^{2} \text { and } \\
h\left(x_{i}\right)= \begin{cases}2 x_{i}-\sin \left(0.5 f_{1} \pi\right) \cos \left(2 \pi f_{1}+i \pi / n\right)-1, & i \text { is even, } \\
2 x_{i}-\cos \left(0.5 f_{1} \pi\right) \sin \left(\frac{1}{3}\left(2 \pi f_{1}+i \pi / n\right)\right)-1, & i \text { is odd. }\end{cases} \\
\text { PS: } x_{i}= \begin{cases}0.5+0.5 \sin \left(0.5 f_{1} \pi\right) \cos \left(2 \pi f_{1}+i \pi / n\right), & i \text { is even, } \\
0.5+0.5 \cos \left(0.5 f_{1} \pi\right) \sin \left(\frac{1}{3}\left(2 \pi f_{1}+i \pi / n\right)\right), & i \text { is odd. }\end{cases} \\
\text { for } i=3, \ldots, n \text {, and } 0 \leq x_{1}, x_{2} \leq 1 . \\
\text { PF: } f_{2}=1-\sqrt{f_{1}}, 0 \leq f_{1} \leq 1 .\end{array}$ & $\begin{array}{l}\text { PS is a 2-D nonlinear surface. } \\
\text { PF is convex. } \\
\text { two objectives. }\end{array}$ \\
\hline$F 4$ & {$[0,1]$} & $\begin{array}{l}f_{1}(x)=\left(x_{1}+x_{2}\right) / 2, \\
f_{2}(x)=g(x)-f_{1}^{2}, \\
\text { where } g(x)=1+\frac{5}{n-2} \sum_{i=3}^{n} h\left(x_{i}\right)^{2}, \text { and } \\
h\left(x_{i}\right)= \begin{cases}2 x_{i}-f_{1} \cos \left(2 \pi f_{1}+i \pi / n\right)-1, & i \text { is even, } \\
2 x_{i}-f_{1} \sin \left(2 \pi f_{1}+i \pi / n\right)-1, & i \text { is odd. }\end{cases} \\
\text { PS: } x_{i}=\left\{\begin{array}{cc}0.5+0.5 f_{1} \cos \left(2 \pi f_{1}+i \pi / n\right), & i \text { is even, } \\
0.5+0.5 f_{1} \sin \left(2 \pi x_{1}+i \pi / n\right), & i \text { is odd, }\end{array}\right. \\
\text { for } i=3, \ldots, n, \text { and } 0 \leq x_{1}, x_{2} \leq 1 \\
\text { PF: } f_{2}=1-f_{1}^{2}, 0 \leq f_{1} \leq 1\end{array}$ & $\begin{array}{l}\text { PS is a 2-D nonlinear surface. } \\
\text { PF is concave. } \\
\text { two objectives. }\end{array}$ \\
\hline$F 5$ & {$[0,1]$} & $\begin{array}{l}f_{1}(x)=\left(x_{1}+x_{2}\right) / 2, \\
f_{2}(x)=g(x)-f_{1}+\sin \left(2 \pi f_{1}\right) /(2 \pi), \\
\text { where } g(x)=1+\frac{5}{n-2} \sum_{i=3}^{n} h\left(x_{i}\right)^{2}, \text { and } \\
h\left(x_{i}\right)= \begin{cases}2 x_{i}-f_{1} \cos \left(2 \pi f_{1}+i \pi / n\right)-1, & i \text { is even, } \\
2 x_{i}-f_{1} \sin \left(\frac{1}{3}\left(2 \pi f_{1}+i \pi / n\right)\right)-1, & i \text { is odd. }\end{cases} \\
\text { PS: } x_{i}= \begin{cases}0.5+0.5 f_{1} \cos \left(2 \pi f_{1}+i \pi / n\right), & i \text { is even, } \\
0.5+0.5 f_{1} \sin \left(\frac{1}{3}\left(2 \pi x_{1}+i \pi / n\right)\right), & i \text { is odd, }\end{cases} \\
\text { for } i=3, \ldots, n, \text { and } 0 \leq x_{1}, x_{2} \leq 1 . \\
\text { PF: } f_{2}=1-f_{1}+\sin \left(2 \pi f_{1}\right) /(2 \pi), 0 \leq f_{1} \leq 1\end{array}$ & $\begin{array}{l}\text { PS is a 2-D nonlinear surface. } \\
\text { PF is neither concave or convex. } \\
\text { two objectives. }\end{array}$ \\
\hline F6 & {$[0,1]$} & $\begin{array}{l}f_{1}(x)=\left(x_{1}+x_{2}+x_{3}\right) / 3, \\
f_{2}(x)=g(x)-f_{1}^{2}, \\
\text { where } g(x)=1+\frac{5}{n-3} \sum_{i=4}^{n} h\left(x_{i}\right)^{2} \text {, and } \\
h\left(x_{i}\right)= \begin{cases}2 x_{i}-\sin \left(0.5 f_{1} \pi\right) \cos \left(2 \pi f_{1}+i \pi / n\right)-1, & \text { i is even, } \\
2 x_{i}-\cos \left(0.5 f_{1} \pi\right) \sin \left(\frac{1}{3}\left(2 \pi f_{1}+i \pi / n\right)\right)-1 & \text { i is odd. }\end{cases} \\
\text { PS: } x_{i}= \begin{cases}0.5+0.5 \sin \left(0.5 f_{1} \pi\right) \cos \left(2 \pi f_{1}+i \pi / n\right), & \text { i is even, } \\
0.5+0.5 \cos \left(0.5 f_{1} \pi\right) \sin \left(\frac{1}{3}\left(2 \pi f_{1}+i \pi / n\right)\right), & \text { i is odd, }\end{cases} \\
\text { for } i=4, \ldots, n, \text { and } 0 \leq x_{1}, x_{2}, x_{3} \leq 1 . \\
\text { PF: } f_{2}=1-f_{1}^{2}, 0 \leq f_{1} \leq 1\end{array}$ & $\begin{array}{l}\text { PS is a 3-D continuous } \\
\text { nonlinear manifold. } \\
\text { PF is concave. } \\
\text { two objectives. }\end{array}$ \\
\hline$F 7$ & {$[0,1]$} & $\begin{array}{l}f_{1}(x)=g(x) \cos \left(0.25 \pi\left(x_{1}+x_{2}\right)\right) \sin \left(0.5 \pi x_{3}\right), \\
f_{2}(x)=g(x) \cos \left(0.25 \pi\left(x_{1}+x_{2}\right)\right) \cos \left(0.5 \pi x_{3}\right), \\
f_{3}(x)=g(x) \sin \left(0.25 \pi\left(x_{1}+x_{2}\right)\right) \\
\text { where } g(x)=1+\frac{5}{n-3} \sum_{i=4}^{n} h\left(x_{i}\right)^{2}, \\
h\left(x_{i}\right)= \begin{cases}2 x_{i}-\sin (0.5 \pi y) \cos (2 \pi y+i \pi / n)-1, & i \text { is even, } \\
2 x_{i}-\cos (0.5 \pi y) \sin \left(\frac{1}{3}(2 \pi y+i \pi / n)\right)-1, & i \text { is odd, }\end{cases} \\
\text { and } y=\left(x_{1}+x_{2}+x_{3}\right) / 3 . \\
\text { PS: } x_{i}= \begin{cases}0.5+0.5 \sin \left(0.5 f_{1} \pi\right) \cos \left(2 \pi f_{1}+i \pi / n\right), & \text { i is even, } \\
0.5+0.5 \cos \left(0.5 f_{1} \pi\right) \sin \left(\frac{1}{3}\left(2 \pi f_{1}+i \pi / n\right)\right), & \text { i is odd, }\end{cases} \\
\text { for } i=4, \ldots, n, \text { and } 0 \leq x_{1}, x_{2}, x_{3} \leq 1 . \\
\text { PF: } f_{1}=\cos (s) \sin (t), f_{2}=\cos (s) \cos (t), f_{3}=\sin (s), 0 \leq s, t \leq \pi / 2 .\end{array}$ & $\begin{array}{l}\text { PS is a 3-D continuous } \\
\text { nonlinear manifold. } \\
\text { PF is concave. } \\
\text { three objectives. }\end{array}$ \\
\hline
\end{tabular}



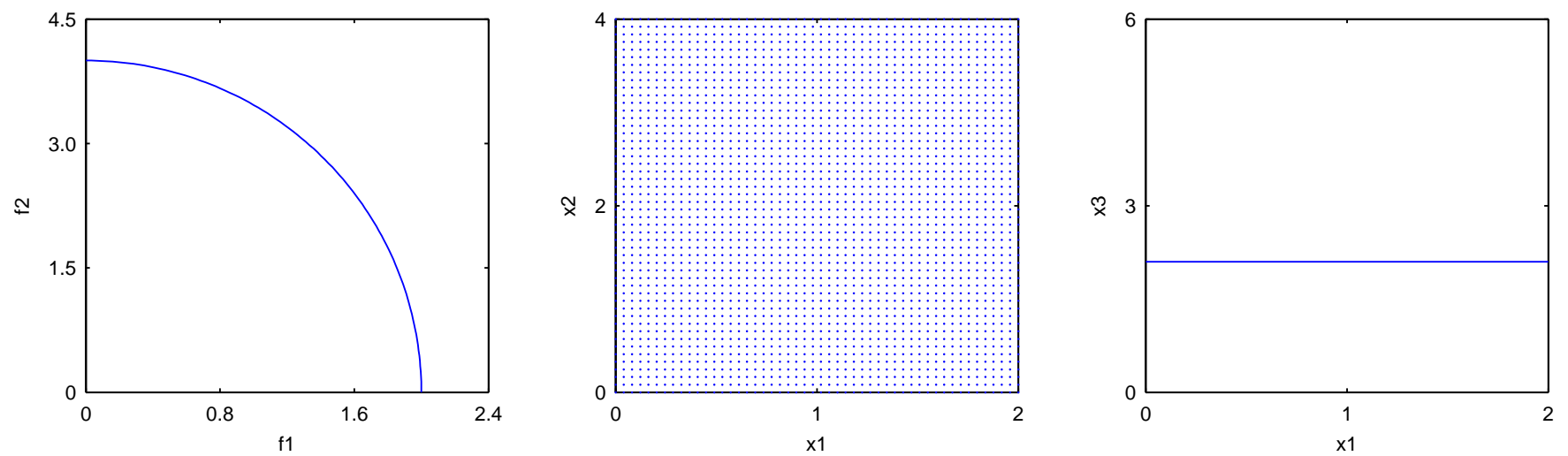

(a) F1
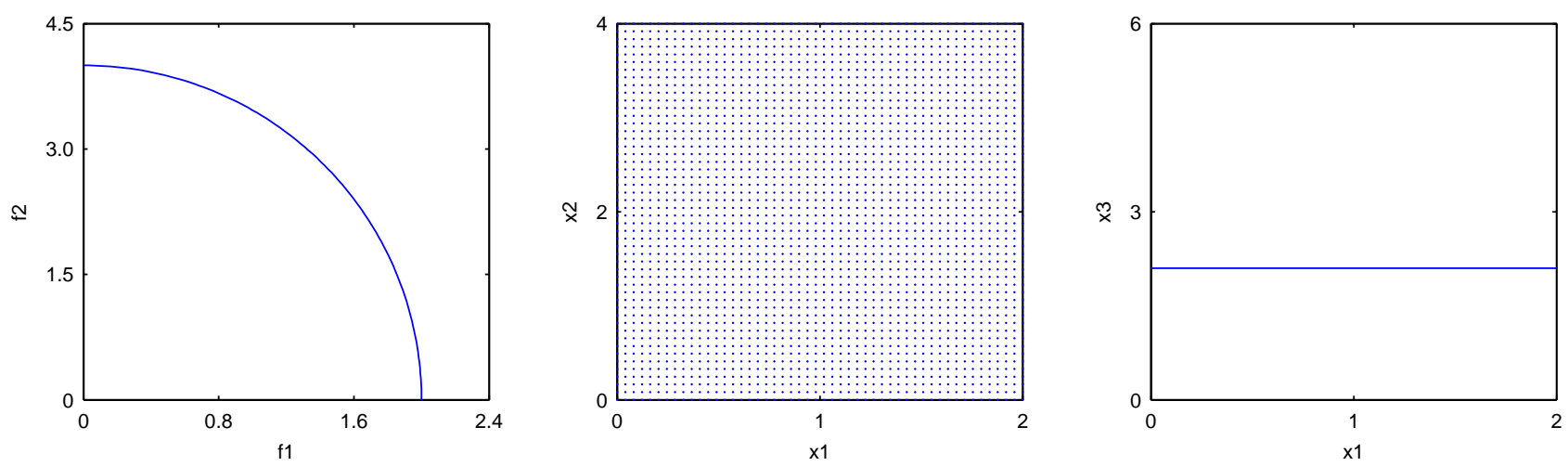

(b) F2
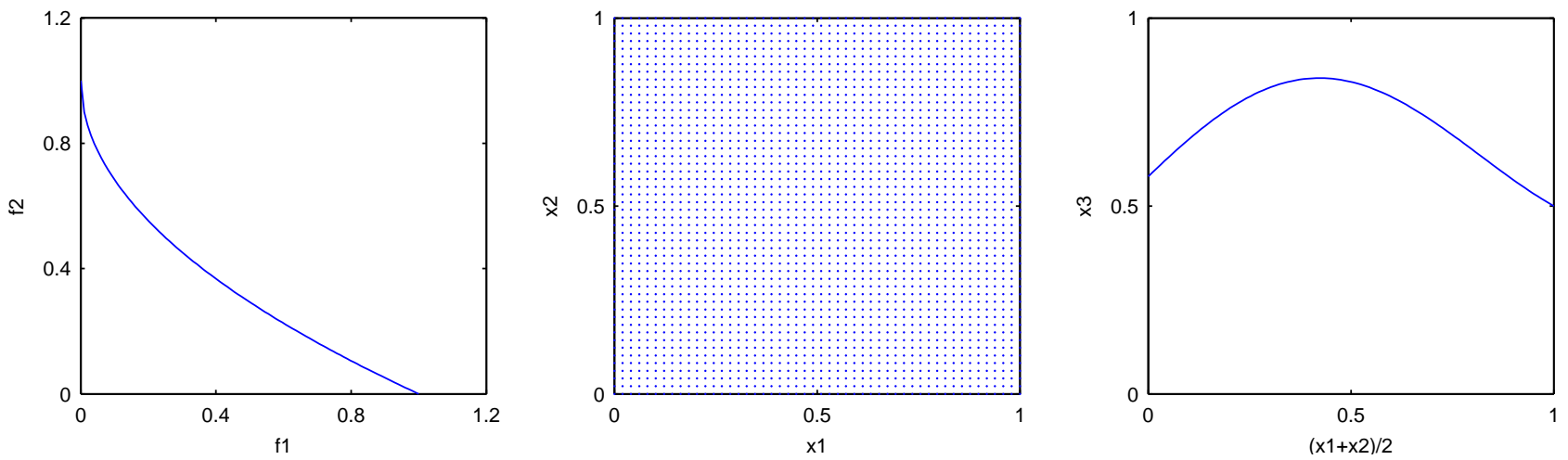

(c) F3
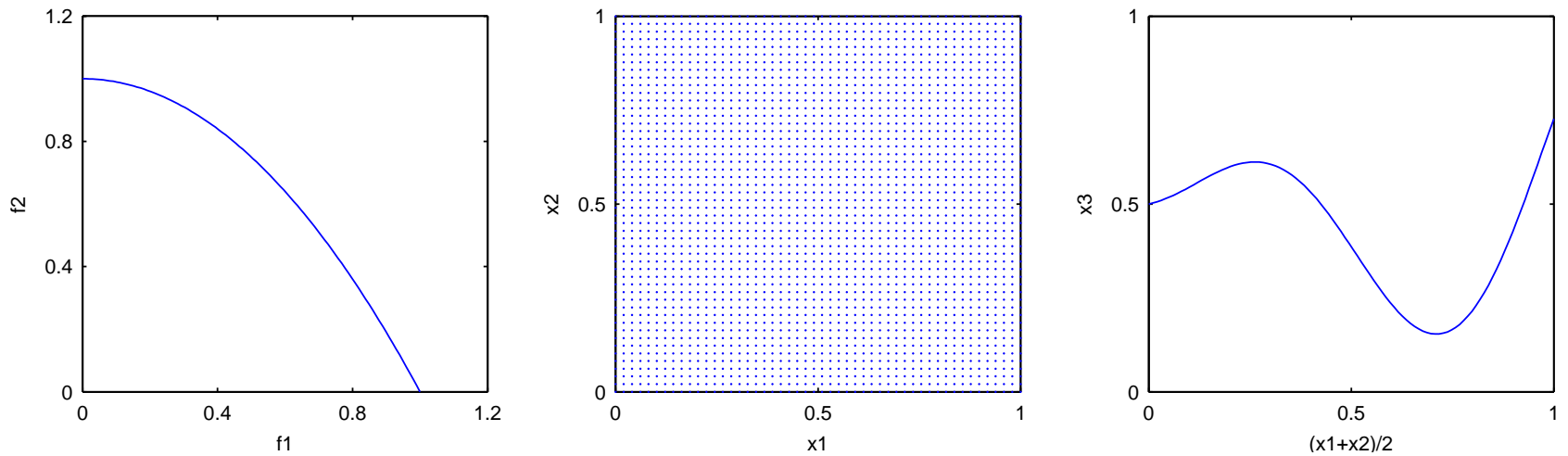

(d) F4

Fig. 2. The PFs and the PSs for F1-F4. (a): F1. (b): F2. (c): F3. (d): F4. Left: the PFs in the objective space. Middle: the projections of the PSs onto the $x_{1}-x_{2}$ space. Right: the projections of the PSs onto the $x_{1}-x_{3}$ space for F1 and F2, and onto the $\frac{x_{1}+x_{2}}{2}-x_{3}$ space for F3 and F4. 

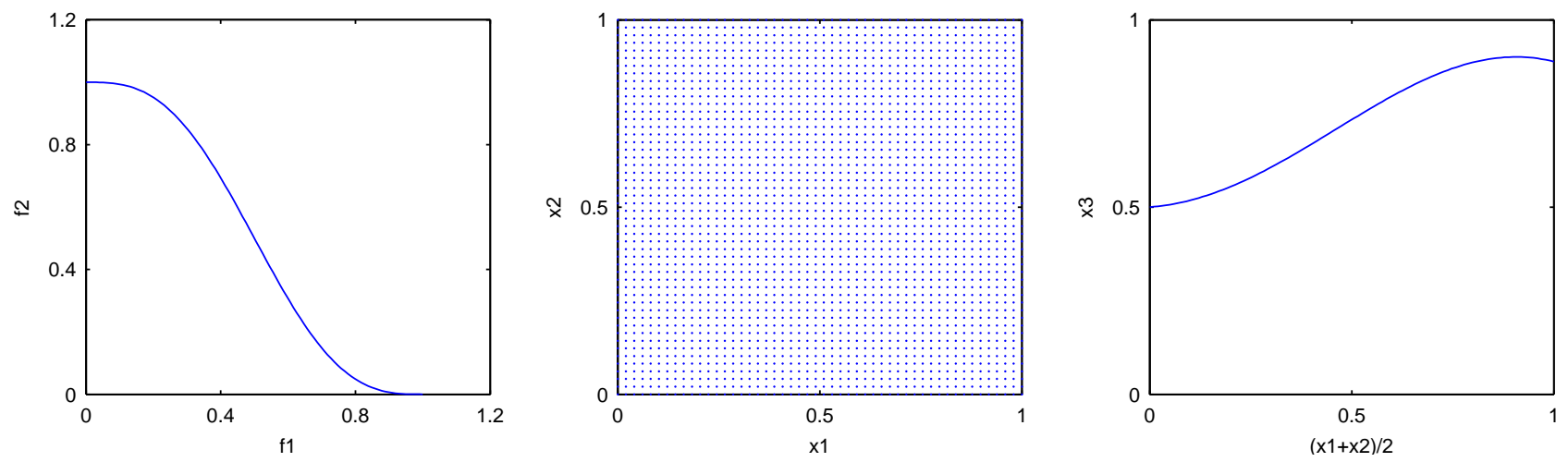

(a) F5
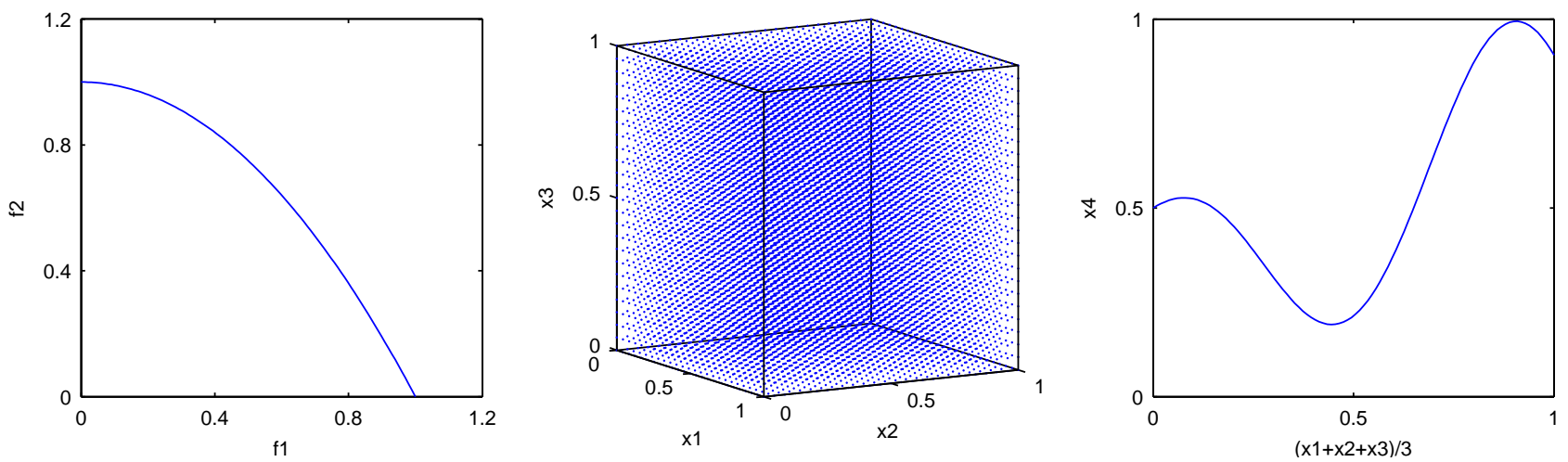

(b) F6
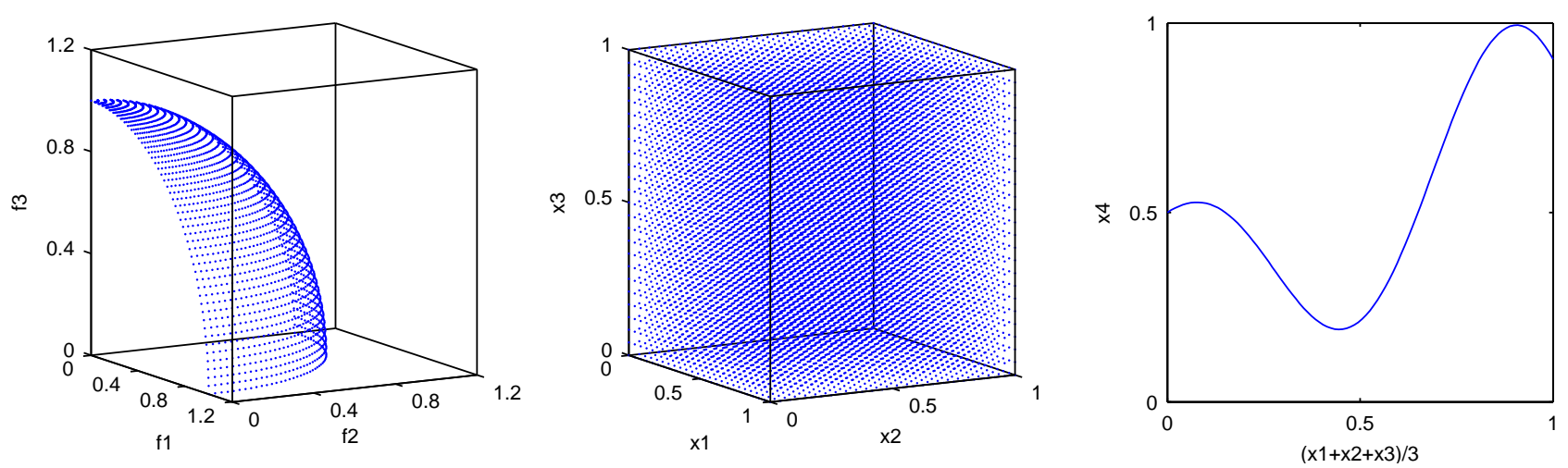

(c) F7

Fig. 3. The PFs and the PSs for F5-F7. (a): F5. (b): F6. (c): F7. Left: PFs in the objective space. Middle: the projections of the PSs onto the $x_{1}-x_{2}$ space for F5, and onto the $x_{1}-x_{2}-x_{3}$ space for F6 and F7. Right: the projections of the PSs onto the $\frac{x_{1}+x_{2}}{2}-x_{3}$ space for F5, and onto the $\frac{x_{1}+x_{2}+x_{3}}{3}-x_{4}$ space for F6 and F7.

\section{B. $F 1-F 2$}

$\mathrm{F} 1$ and $\mathrm{F} 2$ have the same $\mathrm{PS}$, which is a 2-D rectangle parallel to the $x_{1}-x_{2}$ space. The objectives are nonseparable in F1 but separable in F2 [24]. The means and standard deviations can be found in Table III of the $I_{H}^{-}, I G D F$ and $I G D X$ values of the 20 final populations obtained by each algorithm for F1 and F2. Figs. 4 and 5 show, in the objective and decision spaces, the distribution of the final solutions obtained in the runs with the lowest $I G D F$ and $I G D X$ values of each algorithm for these two test instances, respectively.

It is clear from Table III that in terms of the $I G D F$ metric, MMEA is significantly better than the three other algorithms on these two test instances, and in terms of $I_{H}^{-}$metric, MMEA performs better than the three other competitors on F1 but slightly worse than KP1 on F2. The plots in Figs. 4 and 5 show that all the four algorithms can approximate the PF very well and the final populations with the lowest $I G D F$ values obtained by MMEA approximate the PFs slightly better than those obtained Omni-optimizer and KP1. It should be pointed out that Figs. 4 and 5 do not contradict with Table III. Figs. 4 and 5 give the distributions of the final populations with the lowest $I G D$ values while Table III shows the mean/std of the $I G D$ and $I_{H}^{-}$values.

In terms of the $I G D X$ metric, Table III shows that MMEA significantly outperforms the three other algorithms. Actually, one could visually distinguish from Fig. 4 the differences in 

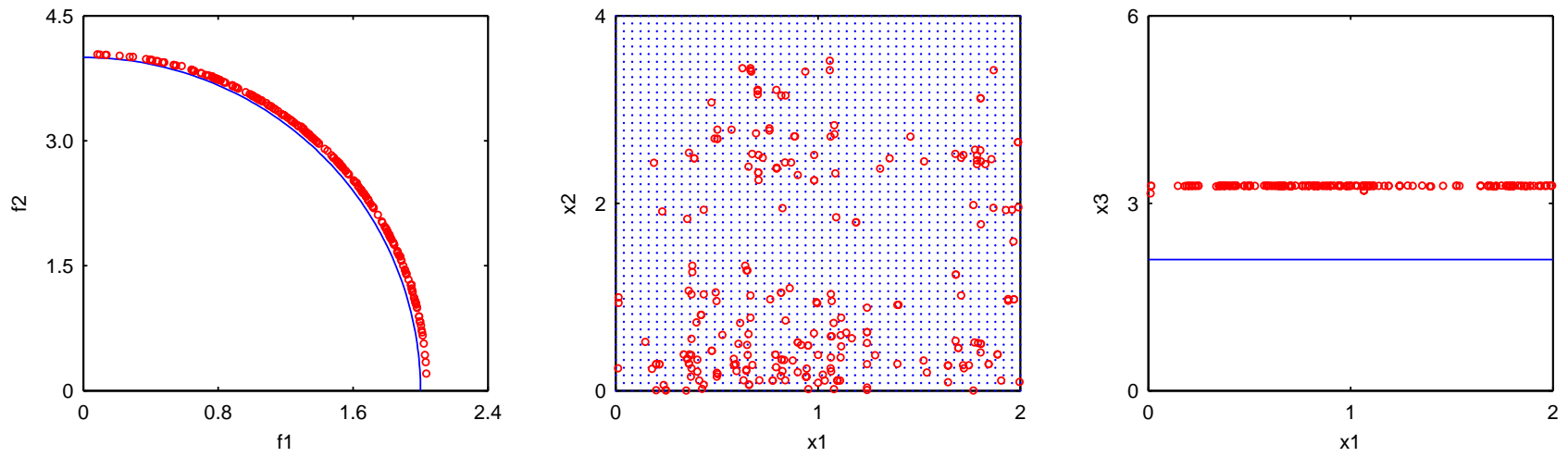

(a) Omni-Optimizer
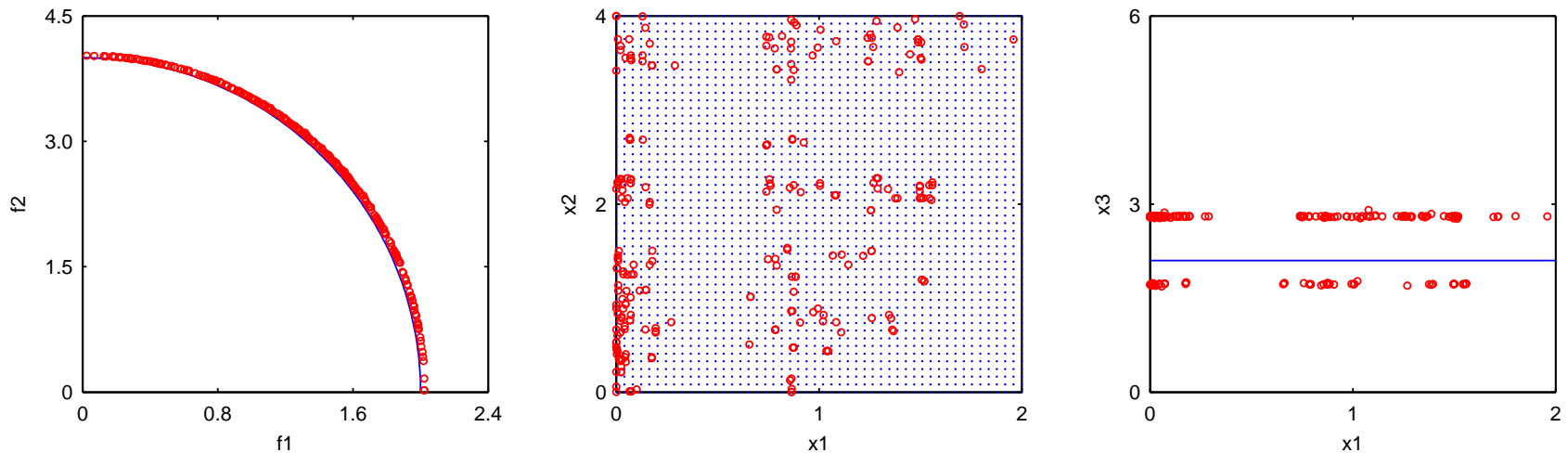

(b) KP1
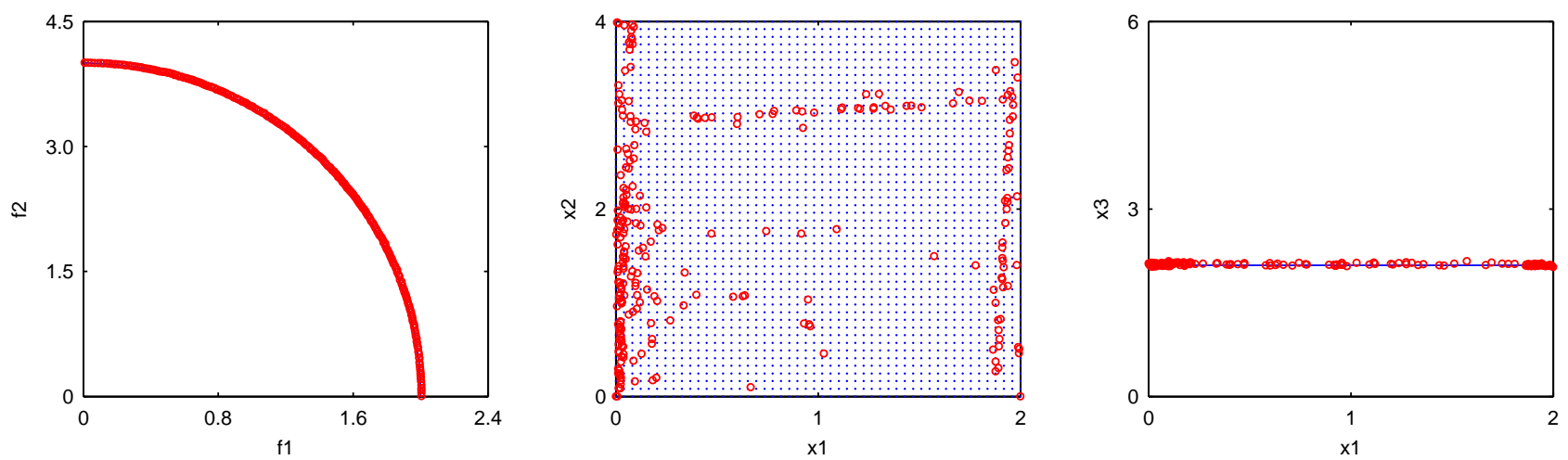

(c) RM-MEDA
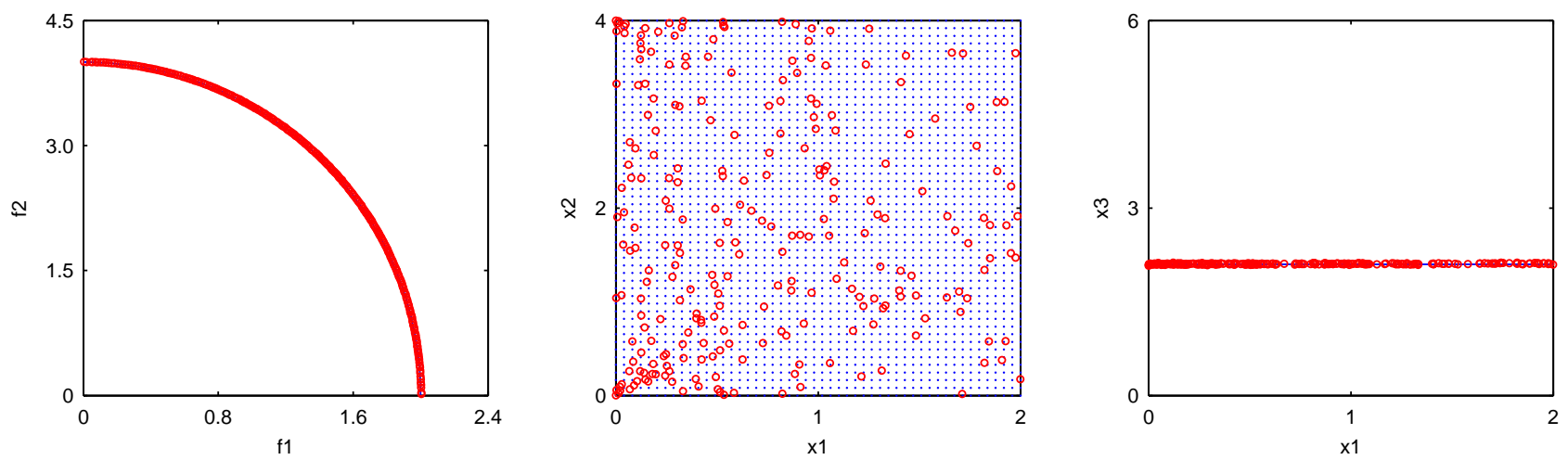

(d) MMEA

Fig. 4. The best approximations obtained by four algorithms for F1: (a) Omni-Optimizer, (b) KP1, (c) RM-MEDA, and (d) MMEA. Left: the distributions of the final solutions in the objective space obtained in the runs with the lowest $I G D F$ values by four respective algorithms. Middle: the distributions of the final solutions in the $x_{1}-x_{2}$ space obtained in the runs with the lowest IGDX values. Right: the distributions of the final solutions in the $x_{1}-x_{3}$ space obtained in the runs with the lowest $I G D X$ values. 

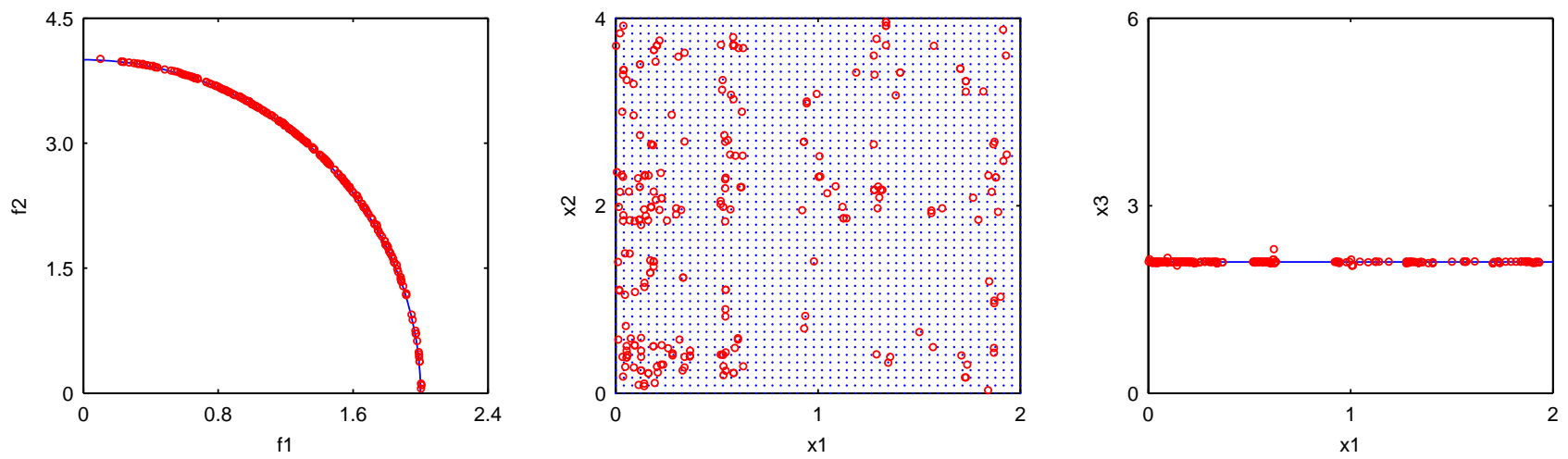

(a) Omni-Optimizer
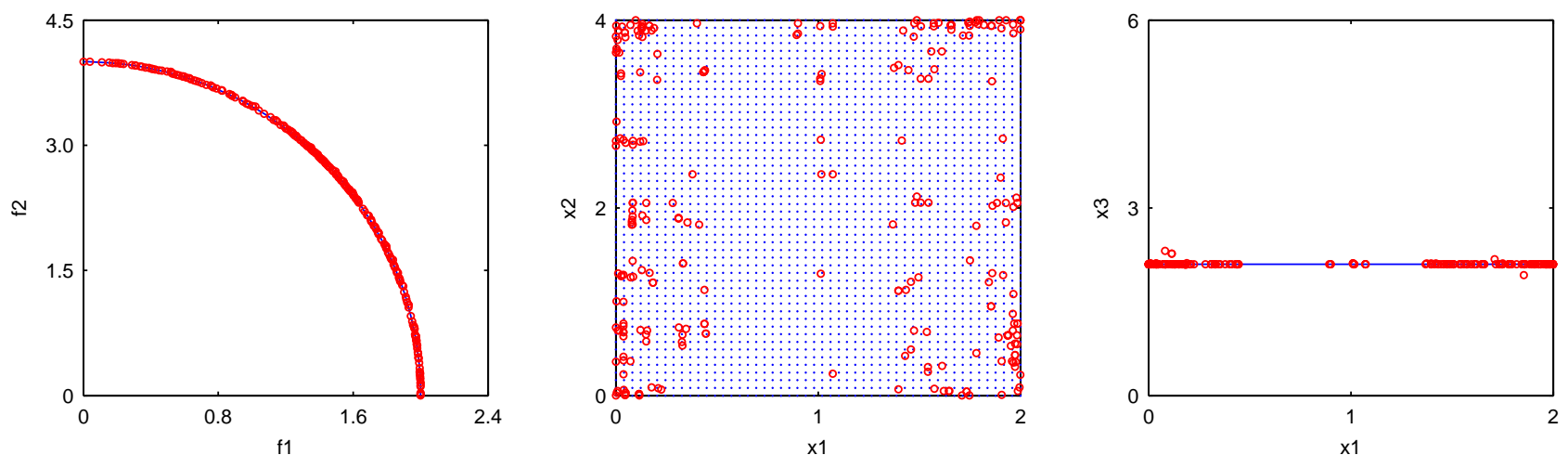

(b) KP1
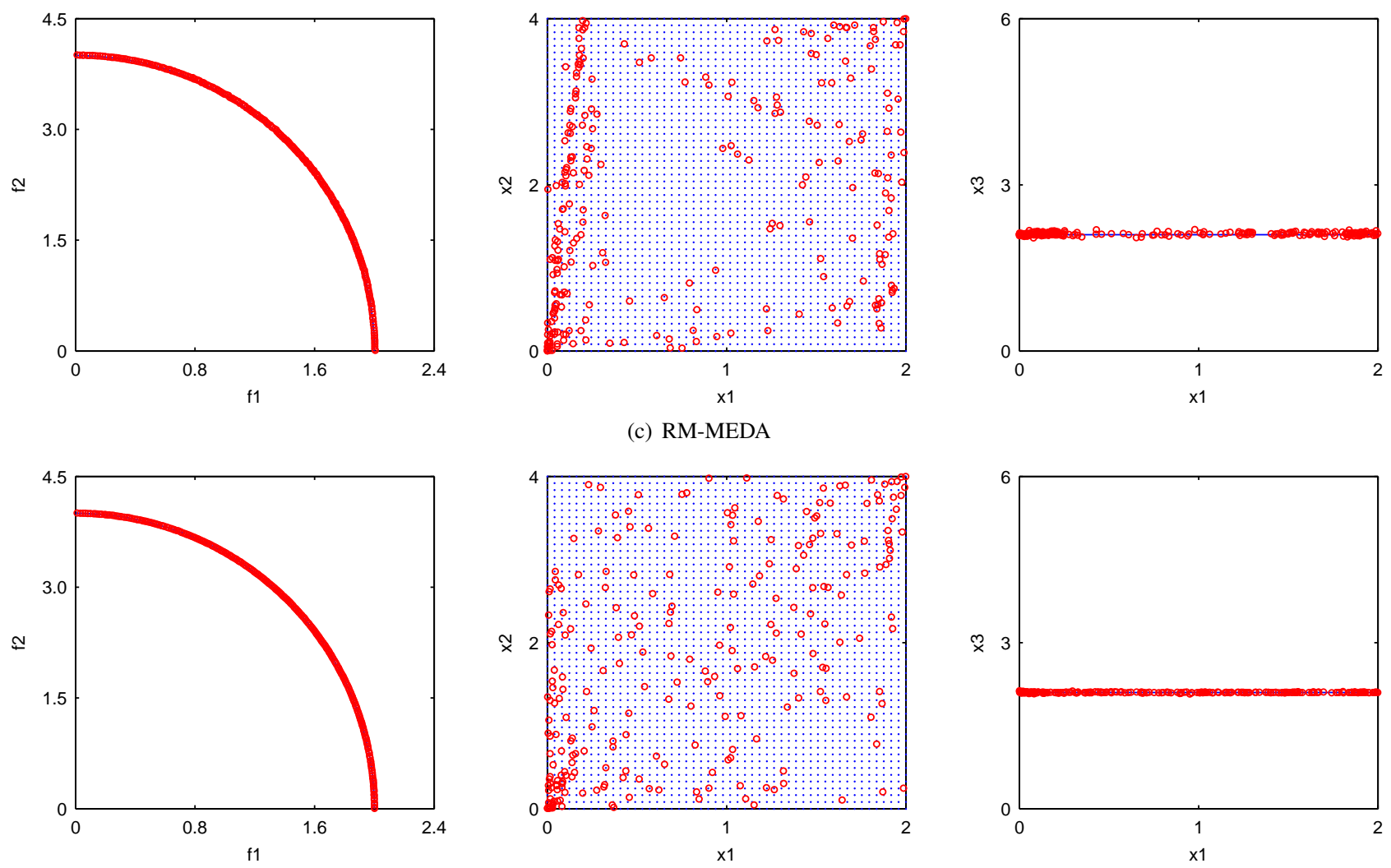

(d) MMEA

Fig. 5. The best approximations obtained by four algorithms for F2: (a) Omni-Optimizer, (b) KP1, (c) RM-MEDA, and (d) MMEA. Left: the distributions of the final solutions in the objective space obtained in the runs with the lowest $I G D F$ values by four respective algorithms. Middle: the distributions of the final solutions in the $x_{1}-x_{2}$ space obtained in the runs with the lowest $I G D X$ values. Right: the distributions of the final solutions in the $x_{1}-x_{3}$ space obtained in the runs with the lowest $I G D X$ values. 
TABLE III

STATISTICAL RESULTS ON F1-F7 ( mean $\pm s t d$.) “+” IN PARENTHESIS: THE ONE-SIDE T-TEST INDICATES THAT THE METRIC VALUE IS LARGER THAN THAT OBTAINED BY MMEA AT THE 95\% SIGNIFICANCE LEVEL. “-” IN PARENTHESIS MEANS A FAILURE IN THE T-TEST TO REJECT THE NULL HYPOTHESIS. IN THE ONE-SIDE T-TEST, THE NULL HYPOTHESIS IS THAT BOTH METRICS VALUES IN TEST ARE FROM THE SAME NORMAL DISTRIBUTION, AND THE ALTERNATIVE HYPOTHESIS IS THAT THE METRIC VALUE IN MMEA IS SMALLER THAN THAT OBTAINED BY ANOTHER ALGORITHM.

\begin{tabular}{|c|c|c|c|c|c|}
\hline & & OMNI & KP1 & RM-MEDA & MMEA \\
\hline \multirow{3}{*}{$\mathrm{F} 1$} & $I H^{-}$ & $0.4653 \pm 0.0867(+)$ & $0.3364 \pm 0.0699(+)$ & $0.0598 \pm 0.0048(+)$ & $\mathbf{0 . 0 4 5 2} \pm 0.0127$ \\
\hline & $I G D F$ & $0.0719 \pm 0.0150(+)$ & $0.0606 \pm 0.0119(+)$ & $0.0110 \pm 0.0008(+)$ & $\mathbf{0 . 0 0 7 9} \pm 0.0016$ \\
\hline & $I G D X$ & $26.7926 \pm 4.6930(+)$ & $23.9950 \pm 7.4775(+)$ & $0.4967 \pm 0.0984(+)$ & $\mathbf{0 . 2 3 2 1} \pm 0.0683$ \\
\hline \multirow{3}{*}{$\mathrm{F} 2$} & $I H^{-}$ & $0.2760 \pm 0.0931(+)$ & $\mathbf{0 . 0 2 8 8} \pm 0.0012(-)$ & $0.0557 \pm 0.0041(+)$ & $0.0298 \pm 0.0044$ \\
\hline & $I G D F$ & $0.0356 \pm 0.0139(+)$ & $0.0108 \pm 0.0009(+)$ & $0.0101 \pm 0.0005(+)$ & $\mathbf{0 . 0 0 6 5} \pm 0.0004$ \\
\hline & $I G D X$ & $0.3174 \pm 0.0336(+)$ & $0.2759 \pm 0.0325(+)$ & $0.4293 \pm 0.0415(+)$ & $\mathbf{0 . 2 1 5 9} \pm 0.0430$ \\
\hline \multirow{3}{*}{ F3 } & $I H^{-}$ & $0.5412 \pm 0.0095(+)$ & $0.0842 \pm 0.0241(+)$ & $\mathbf{0 . 0 0 5 5} \pm 0.0039(-)$ & $0.0059 \pm 0.0042$ \\
\hline & $I G D F$ & $0.5609 \pm 0.0154(+)$ & $0.0776 \pm 0.0290(+)$ & $\mathbf{0 . 0 0 2 6} \pm 0.0018(-)$ & $0.0029 \pm 0.0019$ \\
\hline & $I G D X$ & $1.0458 \pm 0.0134(+)$ & $0.4078 \pm 0.0752(+)$ & $0.2497 \pm 0.0195(+)$ & $\mathbf{0 . 1 0 7 3} \pm 0.0100$ \\
\hline \multirow{3}{*}{$\mathrm{F} 4$} & $I H^{-}$ & $0.4220 \pm 0.0708(+)$ & $0.1920 \pm 0.1213(+)$ & $0.0760 \pm 0.0631(+)$ & $\mathbf{0 . 0 4 0 8} \pm 0.0685$ \\
\hline & $I G D F$ & $0.3310 \pm 0.1038(+)$ & $0.1146 \pm 0.1040(+)$ & $0.0304 \pm 0.0319(-)$ & $\mathbf{0 . 0 1 7 9} \pm 0.0392$ \\
\hline & $I G D X$ & $0.8415 \pm 0.1613(+)$ & $0.4421 \pm 0.2019(+)$ & $0.2795 \pm 0.0544(+)$ & $\mathbf{0 . 1 2 9 3} \pm 0.0892$ \\
\hline \multirow{3}{*}{ F5 } & $I H^{-}$ & $0.3661 \pm 0.1045(+)$ & $0.0297 \pm 0.0042(+)$ & $0.0122 \pm 0.0084(+)$ & $\mathbf{0 . 0 0 8 0} \pm 0.0043$ \\
\hline & $I G D F$ & $0.2677 \pm 0.0973(+)$ & $0.0300 \pm 0.0032(+)$ & $0.0145 \pm 0.0072(+)$ & $\mathbf{0 . 0 0 9 7} \pm 0.0045$ \\
\hline & $I G D X$ & $0.6291 \pm 0.1176(+)$ & $0.2512 \pm 0.0278(+)$ & $0.2149 \pm 0.0322(+)$ & $\mathbf{0 . 0 9 1 8} \pm 0.0105$ \\
\hline \multirow{3}{*}{ F6 } & $I H^{-}$ & $0.2381 \pm 0.0891(+)$ & $0.1757 \pm 0.0291(+)$ & $\mathbf{0 . 0 0 2 5} \pm 0.0003(-)$ & $0.0068 \pm 0.0016$ \\
\hline & $I G D F$ & $0.1366 \pm 0.0707(+)$ & $0.0887 \pm 0.0217(+)$ & $\mathbf{0 . 0 0 1 2} \pm 0.0001(-)$ & $0.0039 \pm 0.0009$ \\
\hline & $I G D X$ & $0.5760 \pm 0.1501(+)$ & $0.4484 \pm 0.0512(+)$ & $0.3405 \pm 0.0240(+)$ & $\mathbf{0 . 1 6 1 6} \pm 0.0275$ \\
\hline \multirow{3}{*}{ F7 } & $I H^{-}$ & $0.6055 \pm 0.0816(+)$ & $0.9028 \pm 0.0000(+)$ & $\mathbf{0 . 0 8 6 6} \pm 0.0046(-)$ & $0.1055 \pm 0.0056$ \\
\hline & $I G D F$ & $0.6073 \pm 0.0869(+)$ & $0.7451 \pm 0.0000(+)$ & $\mathbf{0 . 0 4 7 6} \pm 0.0022(-)$ & $0.0620 \pm 0.0028$ \\
\hline & $I G D X$ & $1.0669 \pm 0.0361(+)$ & $1.3061 \pm 0.0223(+)$ & $\mathbf{0 . 2 1 3 8} \pm 0.0059(-)$ & $0.2387 \pm 0.0092$ \\
\hline
\end{tabular}

approximation quality in the $x_{1}-x_{2}$ and $x_{1}-x_{3}$ spaces between MMEA and the three other methods on F1 and F2: the distributions of the final population found by MMEA are more diverse and uniform that those obtained by the three others. These results indicate that MMEA could tackle MOPs with linear PSs like F1 and F2.

\section{C. $F 3-F 7$}

All these test instances have nonlinear PSs in the decision space. The dimensionality of the PSs of F3-F5 is 2 while that of F6 and F7 is 3.

$I G D F$ and $I^{-}$are for measuring the approximation quality in the decision space. The t-test results in Table III suggest that on F3-F7, in terms of these two metrics, MMEA performs significantly better than KP1 and Omni-Optimizer, but does not always outperform RM-MEDA. It is confirmed to a certain extent by plots in Figs. 6-10: on F3-F6, the final solutions with the lowest $I G D F$ values obtained by MMEA and RM-MEDA approximate the PFs very well while Omni-Optimizer and KP1 always miss part of the PFs; on F7, it is clear that MMEA and RM-MEDA provide better approximations than Omni-Optimizer and KP1, although none could approximate the PF very well. In terms of the $I G D X$ metric, it is evident from Table III that MMEA outperforms the three other algorithms on F3-F7, except RM-MEDA on F7. Figs 6-9 also reveal that the solutions generated by MMEA, are distributed more uniformly in the decision space than those obtained by the three other ones. Table III shows that RM-MEDA slightly outperforms MMEA in terms of all the performance metrics on F7. One cannot tell, however, any big difference in approximation quality between RM-MEDA and MMEA from Fig 10. The reason why MMEA could not outperform RM-MEDA on F7 might be that neither of these two algorithms converges and has not yet started refining their solutions within the given number of function evaluations.

KP1 and Omni-Optimizer promote the population diversity in their selection operators and mainly use the SBX, which was originally proposed for single objective optimization, to generate new solutions. RM-MEDA assumes that the PS dimensionality is $m-1$. MMEA estimates the dimensionality and the shape of the PS and attempts to make the new solutions uniformly distribute around the estimated PS. Our experiments have suggested that reproduction operators are of crucial importance in MOEAs for approximating both the PS and the PF and one should use their problem-specific knowledge in designing reproduction operators in MOEAs. The major reason that KP1 and Omni-Optimizer fail in F3F7 might be that the SBX is not suitable for an MOP with nonlinear PSs. Actually, if two parent solutions are Pareto optimal (i.e. in the PS), it is very likely that their offspring under the SBX are far away from the PS. Since RM-MEDA tries to use an $(m-1)$-D manifold to approximate the PS, the final solutions obtained by it for biobjective test instances, as shown in Figs 6-9, are distributed along 1-D manifolds in the decision space. 

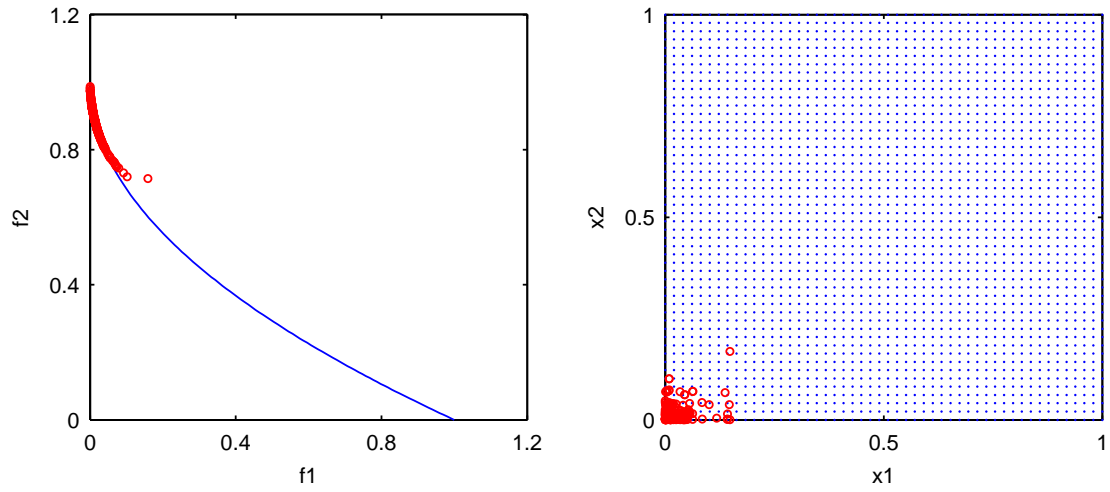

(a) Omni-Optimizer
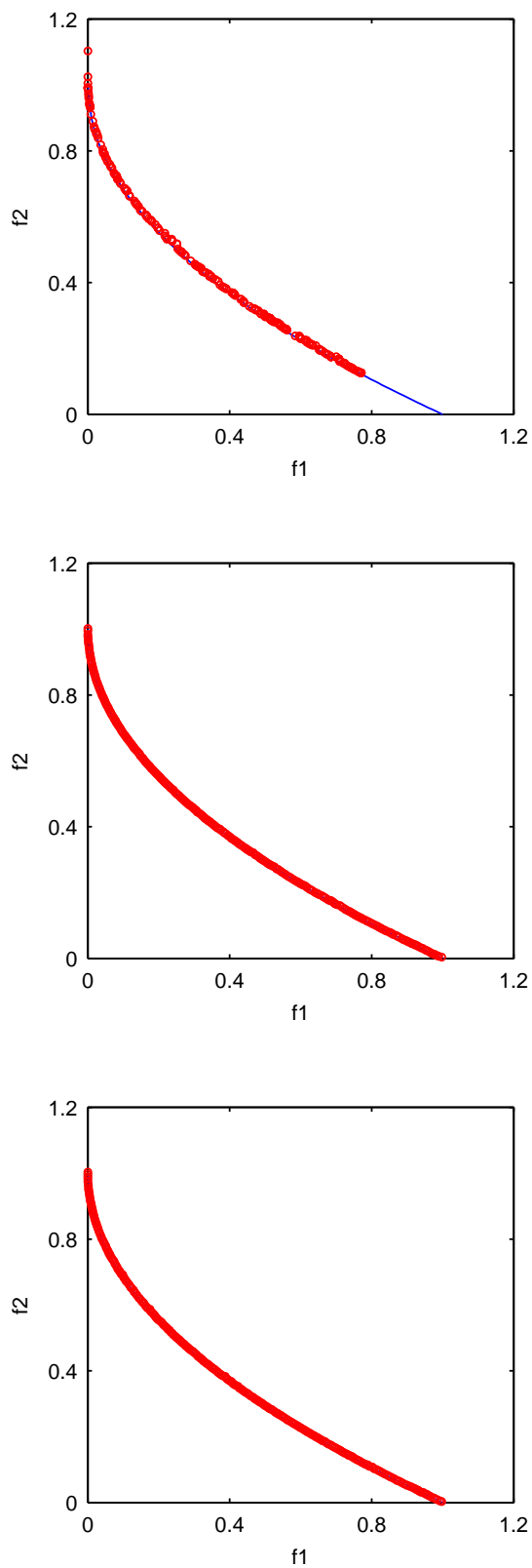

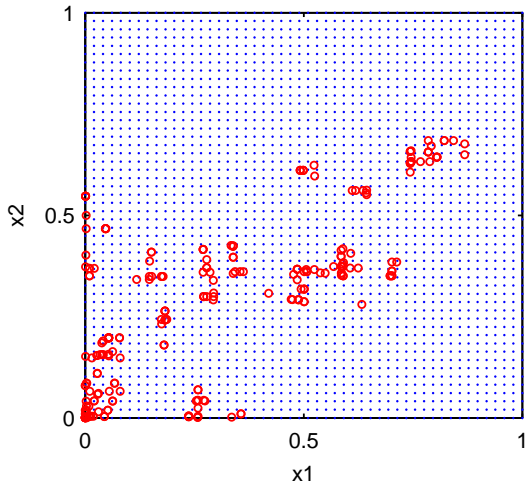

(b) KP1

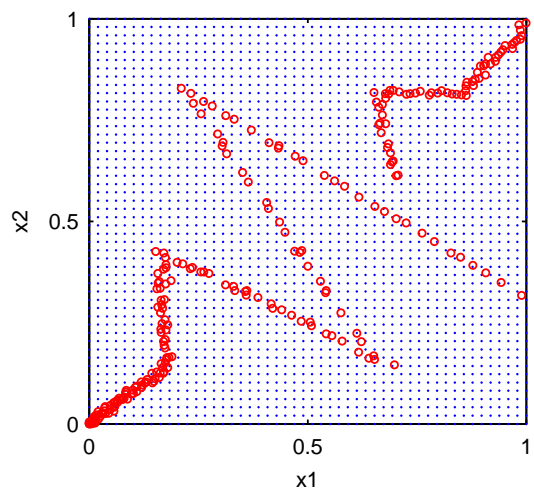

(c) RM-MEDA

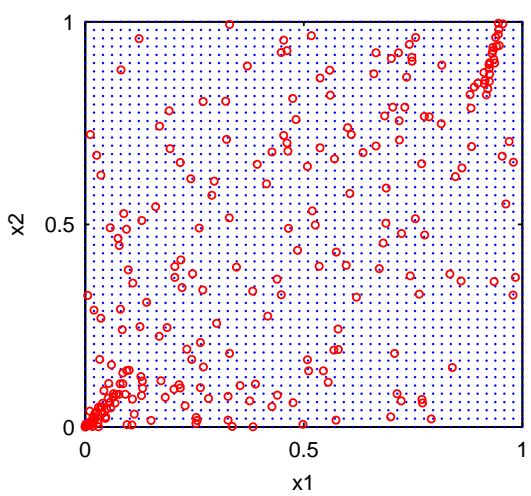

(d) MMEA
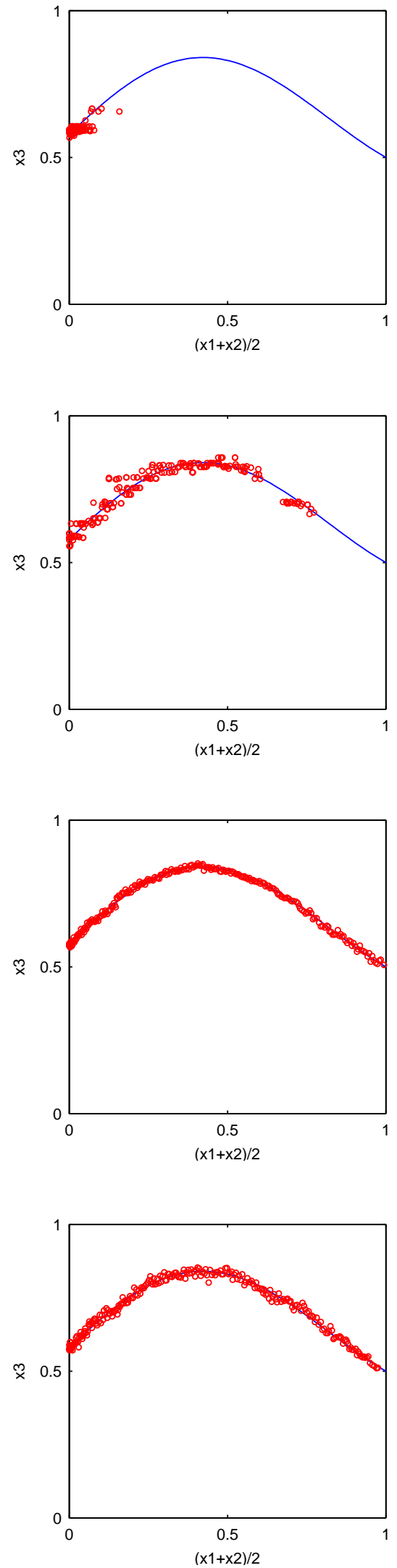

Fig. 6. The best approximations obtained by four algorithms for F3: (a) Omni-Optimizer, (b) KP1, (c) RM-MEDA, and (d) MMEA. Left: the distributions of the final solutions in the objective space obtained in the runs with the lowest $I G D F$ values by three respective algorithms. Middle: the distributions of the final solutions in the $x_{1}-x_{2}$ space obtained in the runs with the lowest $I G D X$ values. Right: the distributions of the final solutions in the $\frac{x_{1}+x_{2}}{2}-x_{3}$ space obtained in the runs with the lowest $I G D X$ values. 

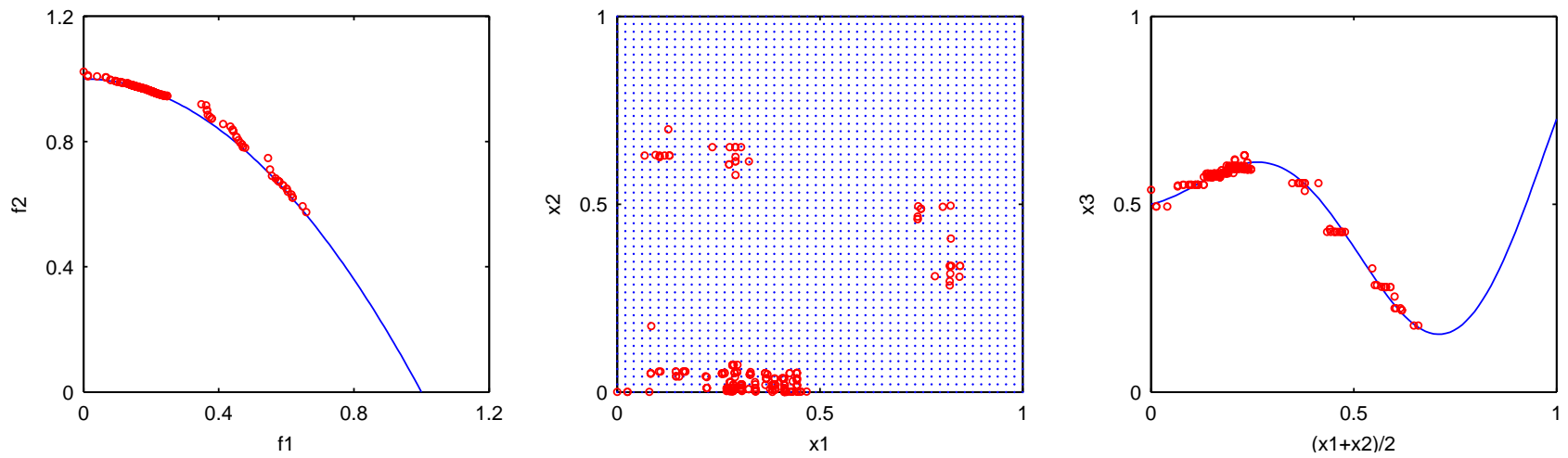

(a) Omni-Optimizer
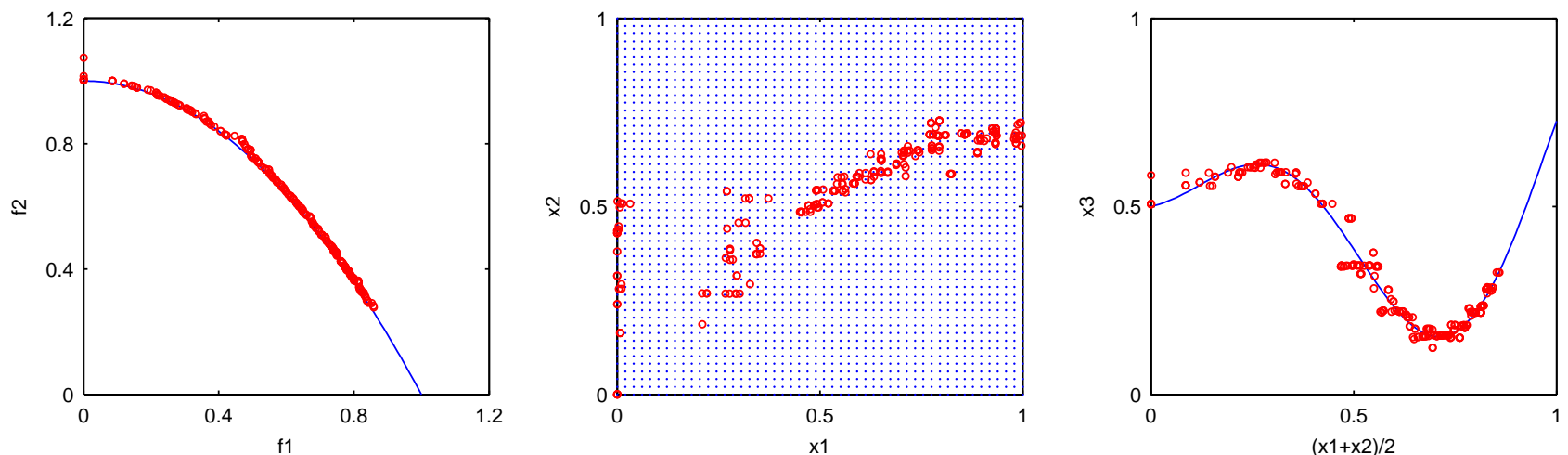

(b) KP1
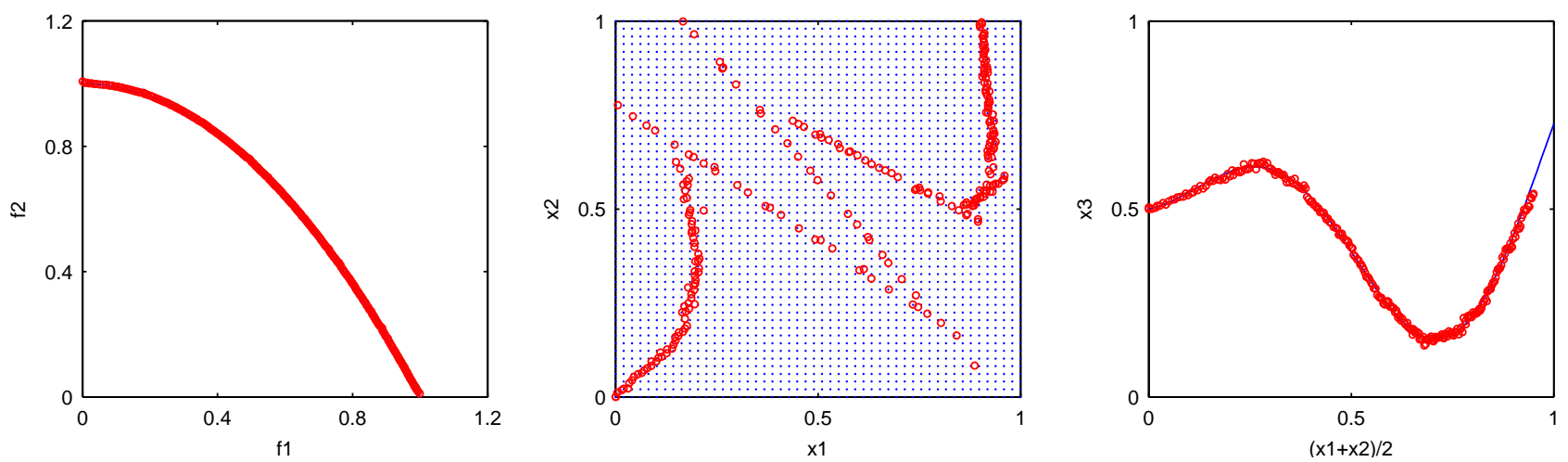

(c) RM-MEDA
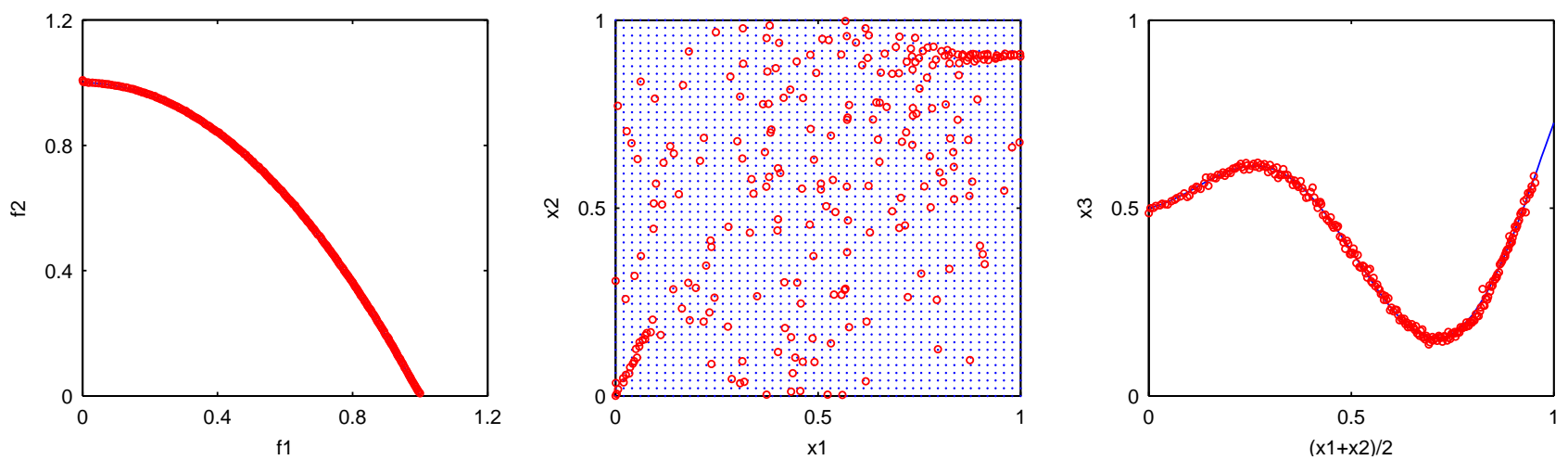

(d) MMEA

Fig. 7. The best approximations obtained by four algorithms for F4: (a) Omni-Optimizer, (b) KP1, (c) RM-MEDA, and (d) MMEA. Left: the distributions of the final solutions in the objective space obtained in the runs with the lowest $I G D F$ values by three respective algorithms. Middle: the distributions of the final solutions in the $x_{1}-x_{2}$ space obtained in the runs with the lowest $I G D X$ values. Right: the distributions of the final solutions in the $\frac{x_{1}+x_{2}}{2}-x_{3}$ space obtained in the runs with the lowest $I G D X$ values. 

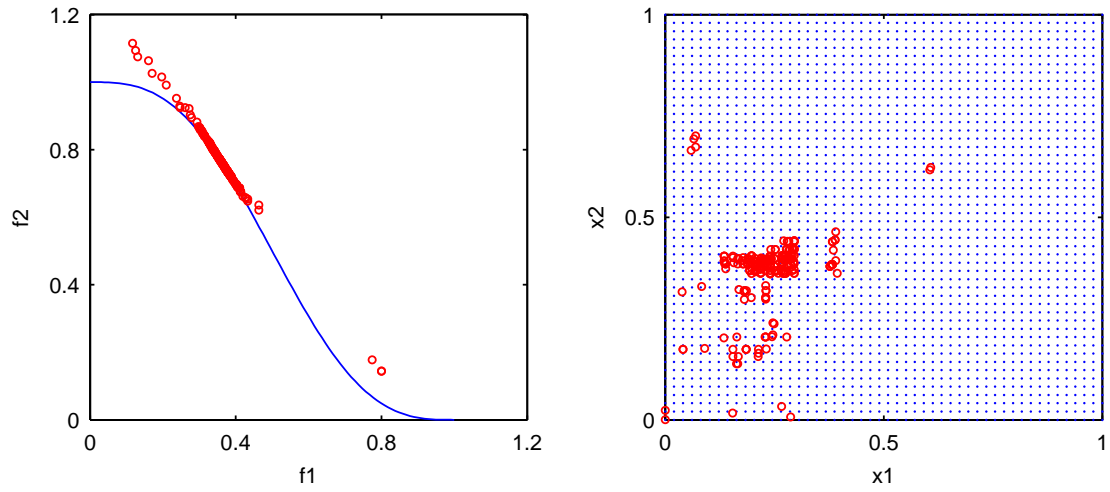

(a) Omni-Optimizer
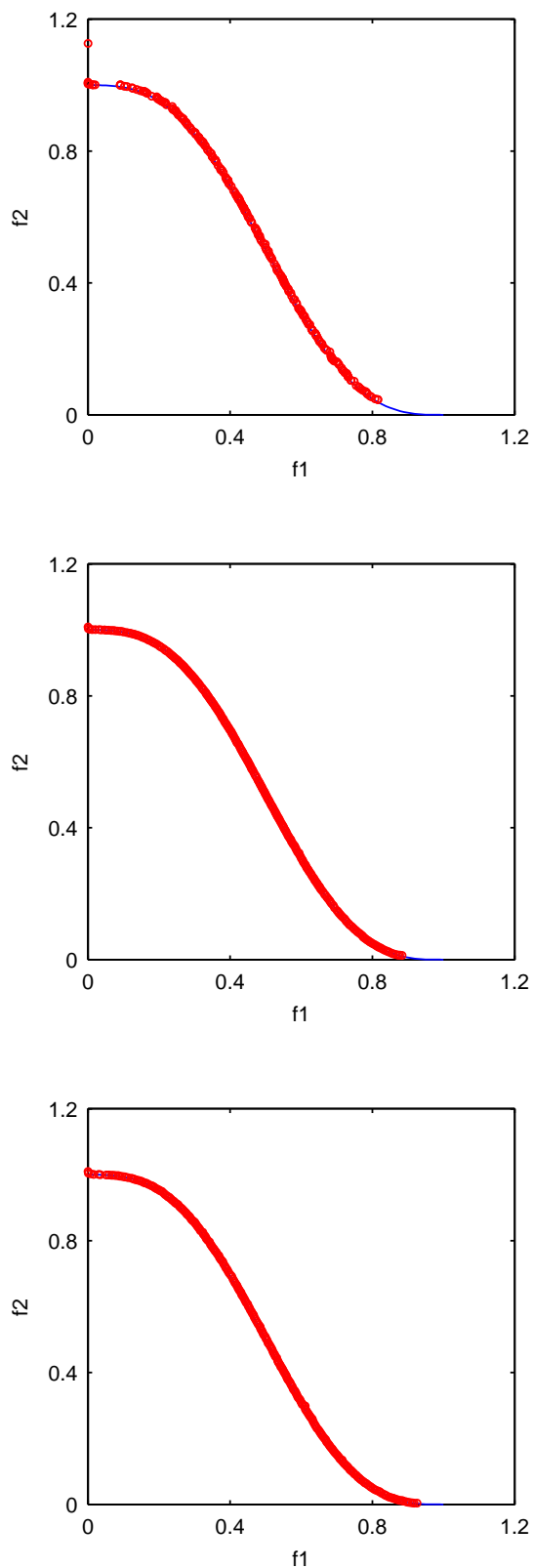

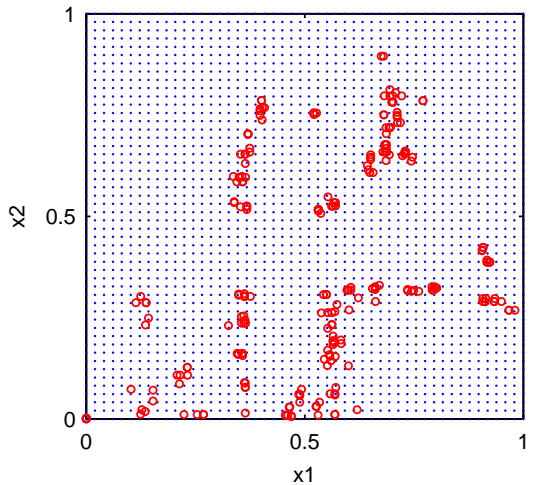

(b) KP1

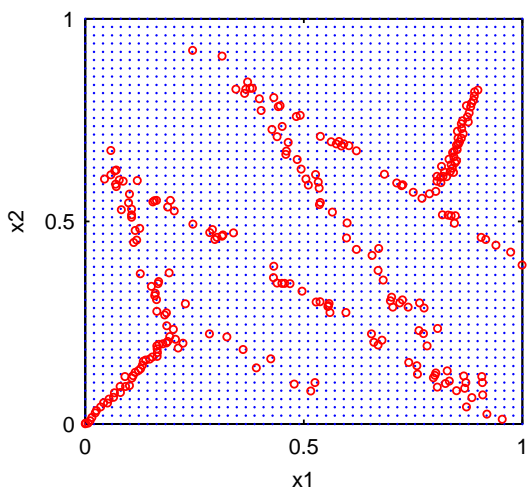

(c) RM-MEDA

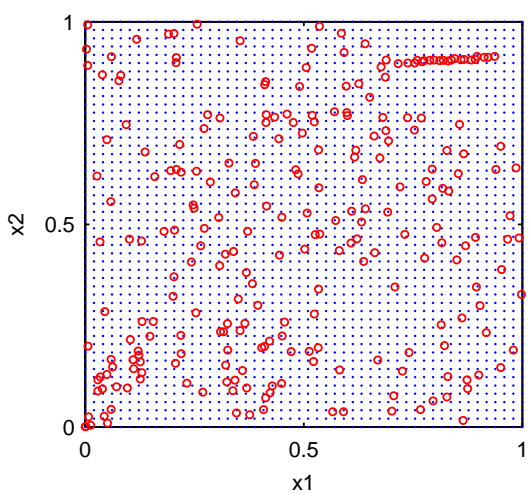

(d) MMEA
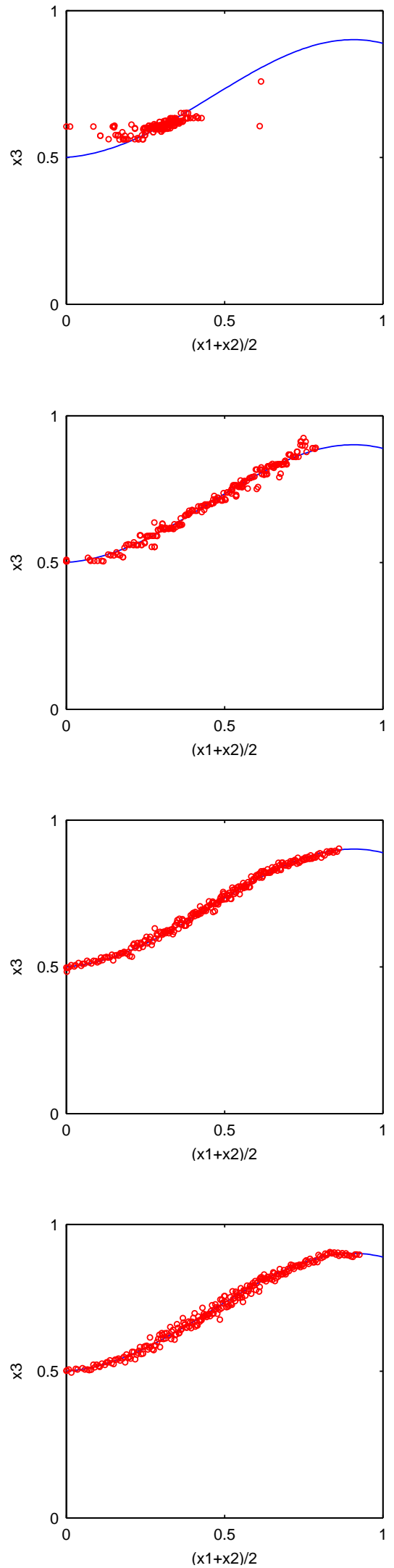

Fig. 8. The best approximations obtained by four algorithms for F5: (a) Omni-Optimizer, (b) KP1, (c) RM-MEDA, and (d) MMEA. Left: the distributions of the final solutions in the objective space obtained in the runs with the lowest $I G D F$ values by three respective algorithms. Middle: the distributions of the final solutions in the $x_{1}-x_{2}$ space obtained in the runs with the lowest $I G D X$ values. Right: the distributions of the final solutions in the $\frac{x_{1}+x_{2}}{2}-x_{3}$ space obtained in the runs with the lowest $I G D X$ values. 

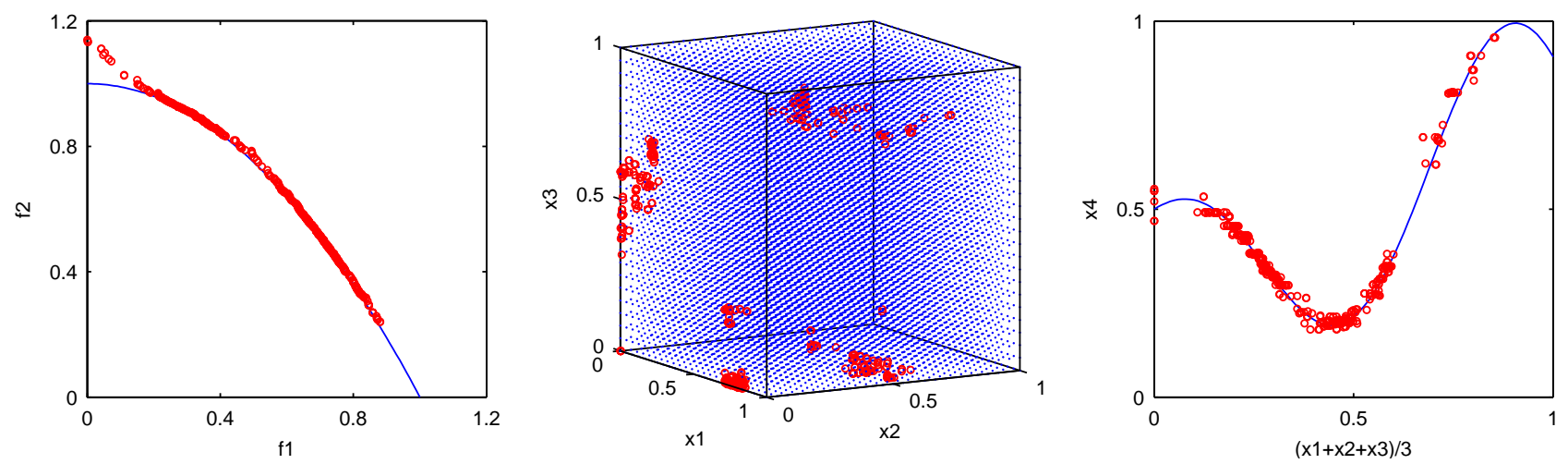

(a) Omni-Optimizer
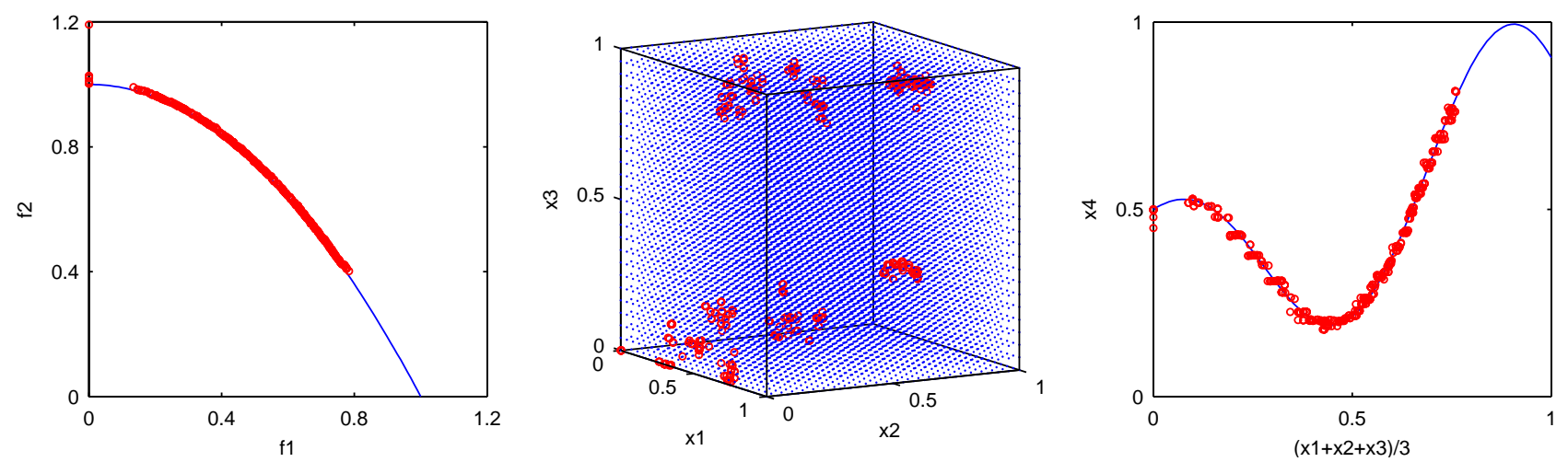

(b) KP1
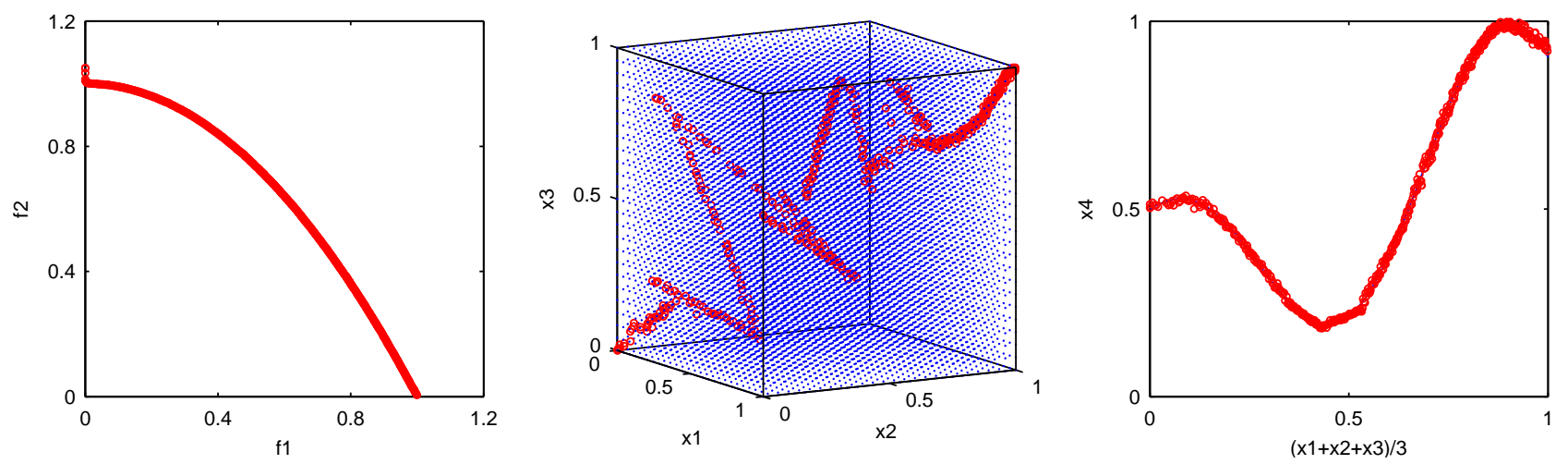

(c) RM-MEDA
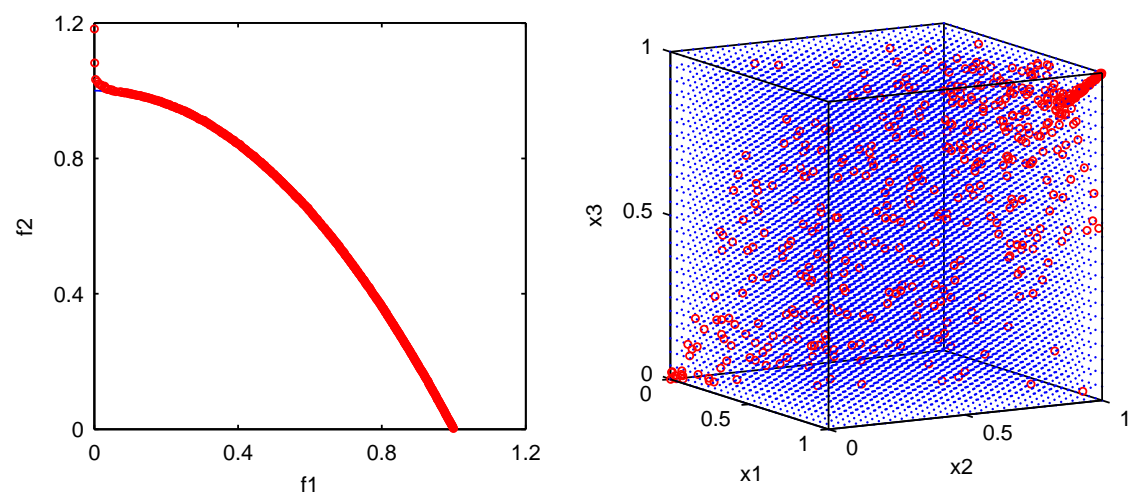

(d) MMEA

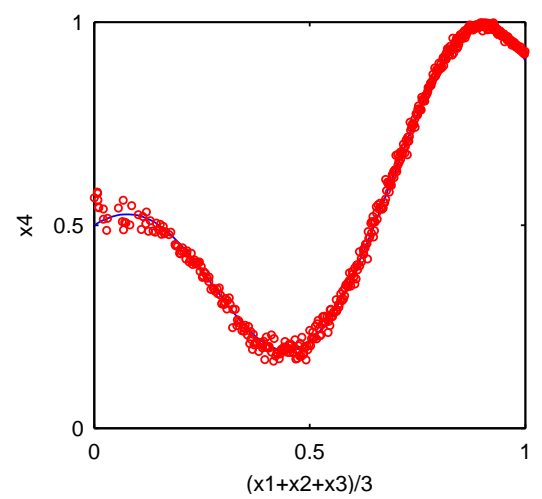

Fig. 9. The best approximations obtained by four algorithms for F6: (a) Omni-Optimizer, (b) KP1, (c) RM-MEDA, and (d) MMEA. Left: the distributions of the final solutions in the objective space obtained in the runs with the lowest $I G D F$ values by three respective algorithms. Middle: the distributions of the final solutions in the $x_{1}-x_{2}-x_{3}$ space obtained in the runs with the lowest $I G D X$ values. Right: the distributions of the final solutions in the $\frac{x_{1}+x_{2}+x_{3}}{3}-x_{4}$ space obtained in the runs with the lowest $I G D X$ values. 

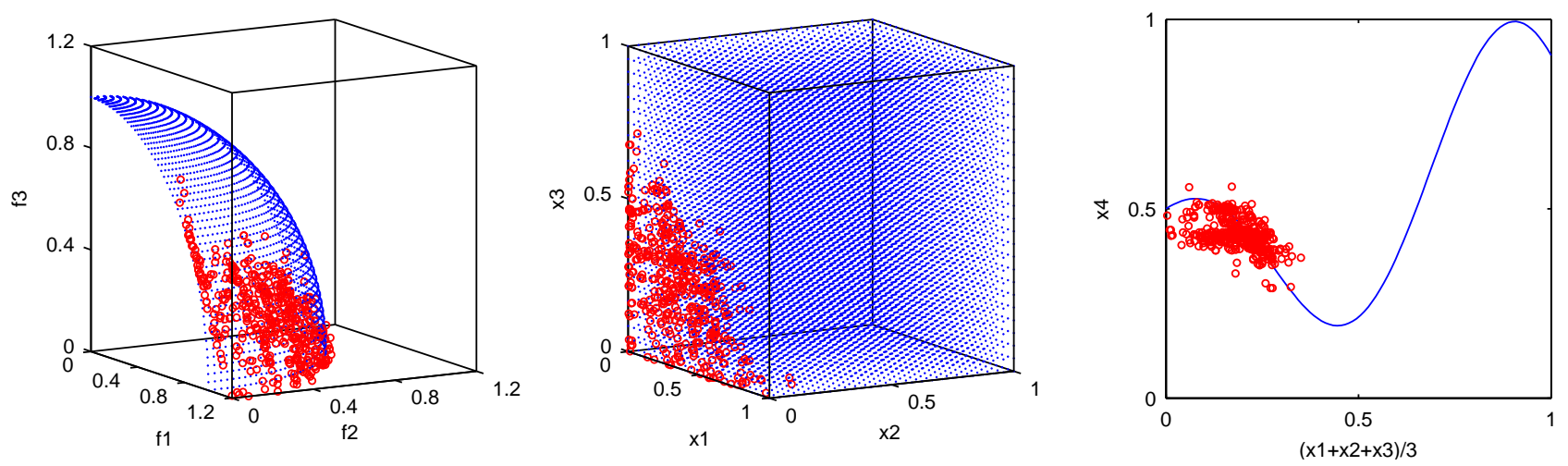

(a) Omni-Optimizer
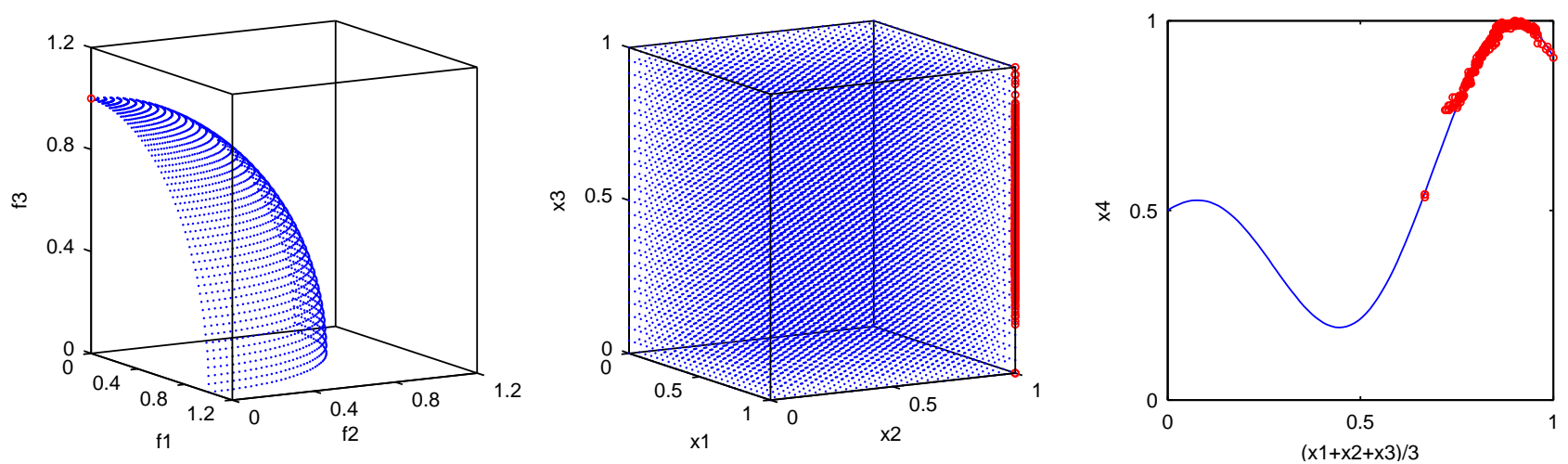

(b) KP1
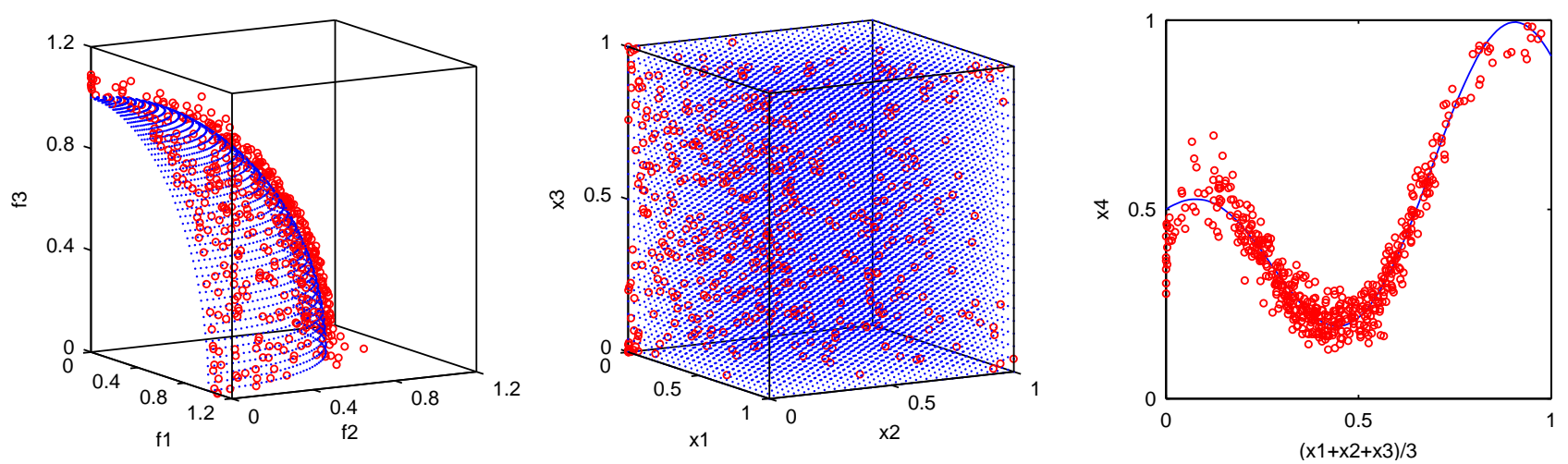

(c) RM-MEDA
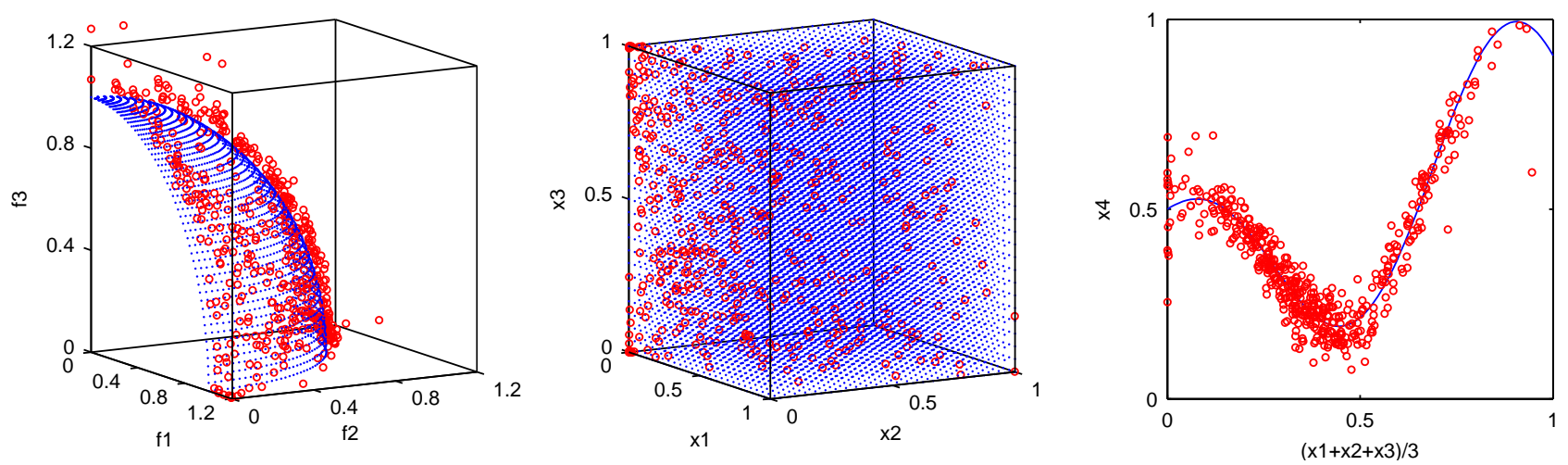

(d) MMEA

Fig. 10. The best approximations obtained by four algorithms for F7: (a) Omni-Optimizer, (b) KP1, (c) RM-MEDA, and (d) MMEA. Left: the distributions of the final solutions in the objective space obtained in the runs with the lowest $I G D F$ values by three respective algorithms. Middle: the distributions of the final solutions in the $x_{1}-x_{2}-x_{3}$ space obtained in the runs with the lowest $I G D X$ values. Right: the distributions of the final solutions in the $\frac{x_{1}+x_{2}+x_{3}}{3}-x_{4}$ space obtained in the runs with the lowest $I G D X$ values. 
TABLE IV

REgular TEST InSTANCES. TheIR PSS AND PFS ARE WITH THE SAME DIMENSIONALITY.

\begin{tabular}{|c|c|c|c|}
\hline Instance & Variables & Objectives & Remarks \\
\hline$Z Z J 08-F 1$ & {$[0,1]^{n}$} & $\begin{array}{l}f_{1}(x)=x_{1} \\
f_{2}(x)=g(x)\left[1-\sqrt{f_{1}(x) / g(x)}\right] \\
g(x)=1+9\left(\sum_{i=2}^{n}\left(x_{i}-x_{1}\right)^{2}\right) /(n-1)\end{array}$ & $\begin{array}{l}\text { convex PF } \\
\text { linear variable linkage }\end{array}$ \\
\hline$Z Z J 08-F 2$ & {$[0,1]^{n}$} & $\begin{array}{l}f_{1}(x)=x_{1} \\
f_{2}(x)=g(x)\left[1-\left(f_{1}(x) / g(x)\right)^{2}\right] \\
g(x)=1+9\left(\sum_{i=2}^{n}\left(x_{i}-x_{1}\right)^{2}\right) /(n-1)\end{array}$ & $\begin{array}{l}\text { concave PF } \\
\text { linear variable linkage }\end{array}$ \\
\hline$Z Z J 08-F 3$ & {$[0,1]^{n}$} & $\begin{array}{l}f_{1}(x)=1-\exp \left(-4 x_{1}\right) \sin ^{6}\left(6 \pi x_{1}\right) \\
f_{2}(x)=g(x)\left[1-\left(f_{1}(x) / g(x)\right)^{2}\right] \\
g(x)=1+9\left[\sum_{i=2}^{n}\left(x_{i}-x_{0}\right)^{2} / 9\right]^{0.25}\end{array}$ & $\begin{array}{l}\text { concave PF } \\
\text { nonuniformly distributed } \\
\text { linear variable linkage }\end{array}$ \\
\hline$Z Z J 08-F 4$ & {$[0,1]^{n}$} & $\begin{array}{l}f_{1}(x)=\cos \left(\frac{\pi}{2} x_{1}\right) \cos \left(\frac{\pi}{2} x_{2}\right)(1+g(x)) \\
f_{2}(x)=\cos \left(\frac{\pi}{2} x_{1}\right) \sin \left(\frac{\pi}{2} x_{2}\right)(1+g(x)) \\
f_{3}(x)=\sin \left(\frac{\pi}{2} x_{1}\right)(1+g(x)) \\
g(x)=\sum_{i=3}^{n}\left(x_{i}-x_{1}\right)^{2}\end{array}$ & $\begin{array}{l}\text { concave PF } \\
\text { linear variable linkage } \\
3 \text { objectives }\end{array}$ \\
\hline$Z Z J 08-F 5$ & {$[0,1]^{n}$} & $\begin{array}{l}f_{1}(x)=x_{1} \\
f_{2}(x)=g(x)\left[1-\sqrt{x_{1} / g(x)}\right] \\
g(x)=1+9\left(\sum_{i=2}^{n}\left(x_{i}^{2}-x_{1}\right)^{2}\right) /(n-1)\end{array}$ & $\begin{array}{l}\text { convex PF } \\
\text { nonlinear variable linkage }\end{array}$ \\
\hline$Z Z J 08-F 6$ & {$[0,1]^{n}$} & $\begin{array}{l}f_{1}(x)=\sqrt{x_{1}} \\
f_{2}(x)=g(x)\left[1-\left(f_{1}(x) / g(x)\right)^{2}\right] \\
g(x)=1+9\left(\sum_{i=2}^{n}\left(x_{i}^{2}-x_{1}\right)^{2}\right) /(n-1)\end{array}$ & $\begin{array}{l}\text { concave PF } \\
\text { nonlinear variable linkage }\end{array}$ \\
\hline$Z Z J 08-F 7$ & {$[0,1]^{n}$} & $\begin{array}{l}f_{1}(x)=1-\exp \left(-4 x_{1}\right) \sin ^{6}\left(6 \pi x_{1}\right) \\
f_{2}(x)=g(x)\left[1-\left(f_{1}(x) / g(x)\right)^{2}\right] \\
g(x)=1+9\left[\sum_{i=2}^{n}\left(x_{i}^{2}-x_{0}\right)^{2} / 9\right]^{0.25}\end{array}$ & $\begin{array}{l}\text { concave PF } \\
\text { nonuniformly distributed } \\
\text { nonlinear variable linkage }\end{array}$ \\
\hline$Z Z J 08-F 8$ & {$[0,1]^{n}$} & $\begin{array}{l}f_{1}(x)=\cos \left(\frac{\pi}{2} x_{1}\right) \cos \left(\frac{\pi}{2} x_{2}\right)(1+g(x)) \\
f_{2}(x)=\cos \left(\frac{\pi}{2} x_{1}\right) \sin \left(\frac{\pi}{2} x_{2}\right)(1+g(x)) \\
f_{3}(x)=\sin \left(\frac{\pi}{2} x_{1}\right)(1+g(x)) \\
g(x)=\sum_{i=3}^{n}\left(x_{i}^{2}-x_{1}\right)^{2}\end{array}$ & $\begin{array}{l}\text { concave } \mathrm{PF} \\
\text { nonlinear variable linkage } \\
3 \text { objectives }\end{array}$ \\
\hline
\end{tabular}
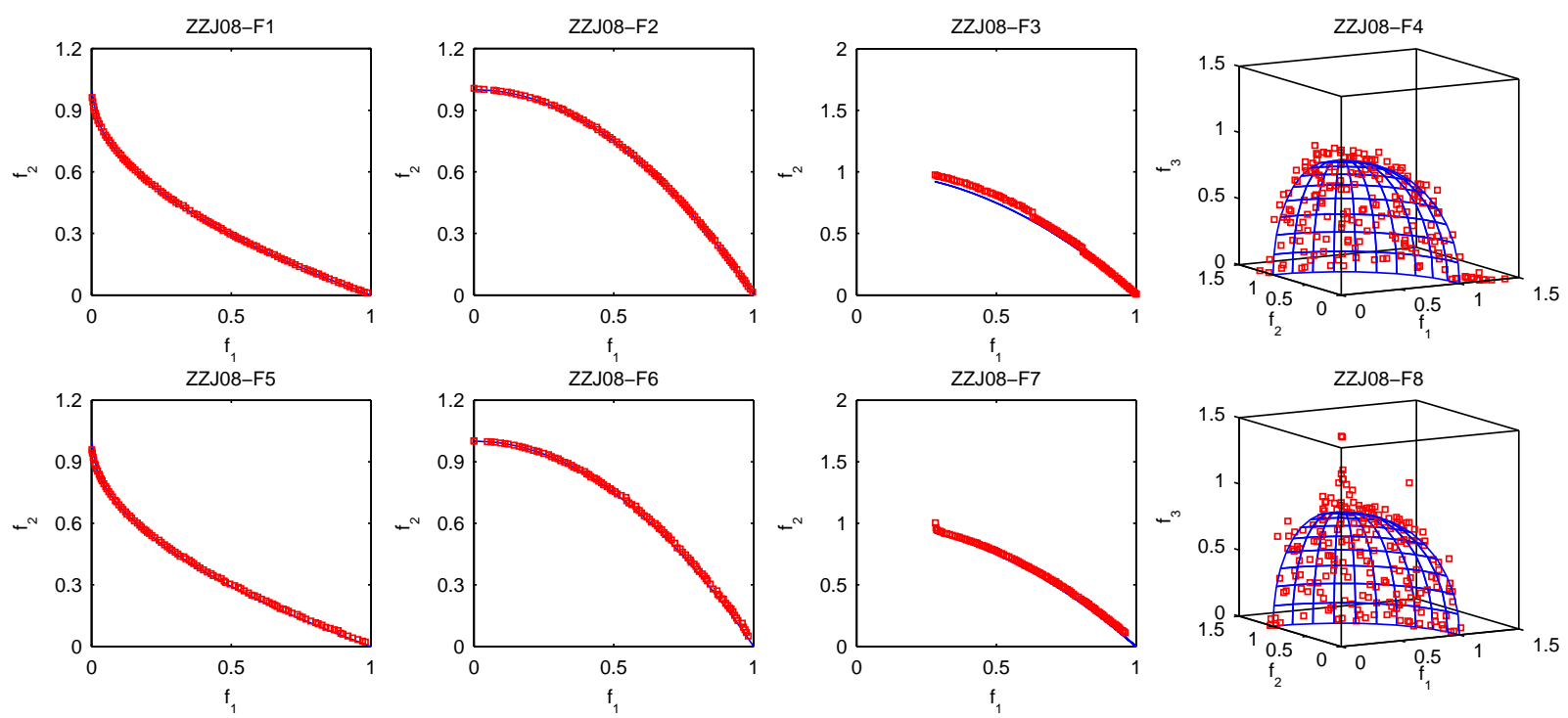

Fig. 11. The approximations with the lowest $I G D F$ values obtained by RM-MEDA on regular MOPs. 
TABLE V

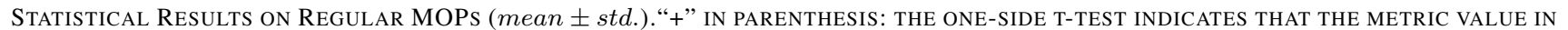
RM-MEDA IS LARGER THAN THAT OBTAINED BY MMEA AT THE 95\% SIGNIFICANCE LEVEL. “-” IN PARENTHESIS MEANS A FAILURE IN THE T-TEST TO REJECT THE NULL HYPOTHESIS.

\begin{tabular}{ccccc}
\hline \hline \multirow{2}{*}{ Instance } & RM-MEDA & MMEA & RM-MEDA & MMEA \\
\hline \hline ZZJ08-F1 & $0.0049 \pm 0.0001(+)$ & $\mathbf{0 . 0 0 4 4} \pm 0.0003$ & $0.0116 \pm 0.0011(+)$ & $\mathbf{0 . 0 0 7 0} \pm 0.0017$ \\
ZZJ08-F2 & $0.0056 \pm 0.0002(+)$ & $\mathbf{0 . 0 0 4 4} \pm 0.0004$ & $0.0190 \pm 0.0029(+)$ & $\mathbf{0 . 0 0 8 4} \pm 0.0032$ \\
ZZJ08-F3 & $0.0094 \pm 0.0037(+)$ & $\mathbf{0 . 0 0 4 7} \pm 0.0005$ & $0.0382 \pm 0.0593(+)$ & $\mathbf{0 . 0 0 7 8} \pm 0.0011$ \\
ZZJ08-F4 & $0.0723 \pm 0.0036(+)$ & $\mathbf{0 . 0 5 2 7} \pm 0.0023$ & $0.1596 \pm 0.0113(+)$ & $\mathbf{0 . 0 8 0 3} \pm 0.0054$ \\
ZZJ08-F5 & $0.0079 \pm 0.0036(-)$ & $\mathbf{0 . 0 0 7 6} \pm 0.0011$ & $0.0234 \pm 0.0130(+)$ & $\mathbf{0 . 0 1 3 5} \pm 0.0026$ \\
ZZJ08-F6 & $0.0136 \pm 0.0183(-)$ & $\mathbf{0 . 0 0 9 3} \pm 0.0034$ & $0.0644 \pm 0.0708(+)$ & $\mathbf{0 . 0 2 7 1} \pm 0.0232$ \\
ZZJ08-F7 & $0.0981 \pm 0.0137(+)$ & $\mathbf{0 . 0 5 8 2} \pm 0.0079$ & $0.1019 \pm 0.0138(+)$ & $\mathbf{0 . 0 7 7 4} \pm 0.0073$ \\
ZZJ08-F8 & $0.0694 \pm 0.0029(-)$ & $\mathbf{0 . 0 6 8 4} \pm 0.0280$ & $0.1211 \pm 0.0096(+)$ & $\mathbf{0 . 1 0 2 6} \pm 0.0188$ \\
\hline \hline
\end{tabular}
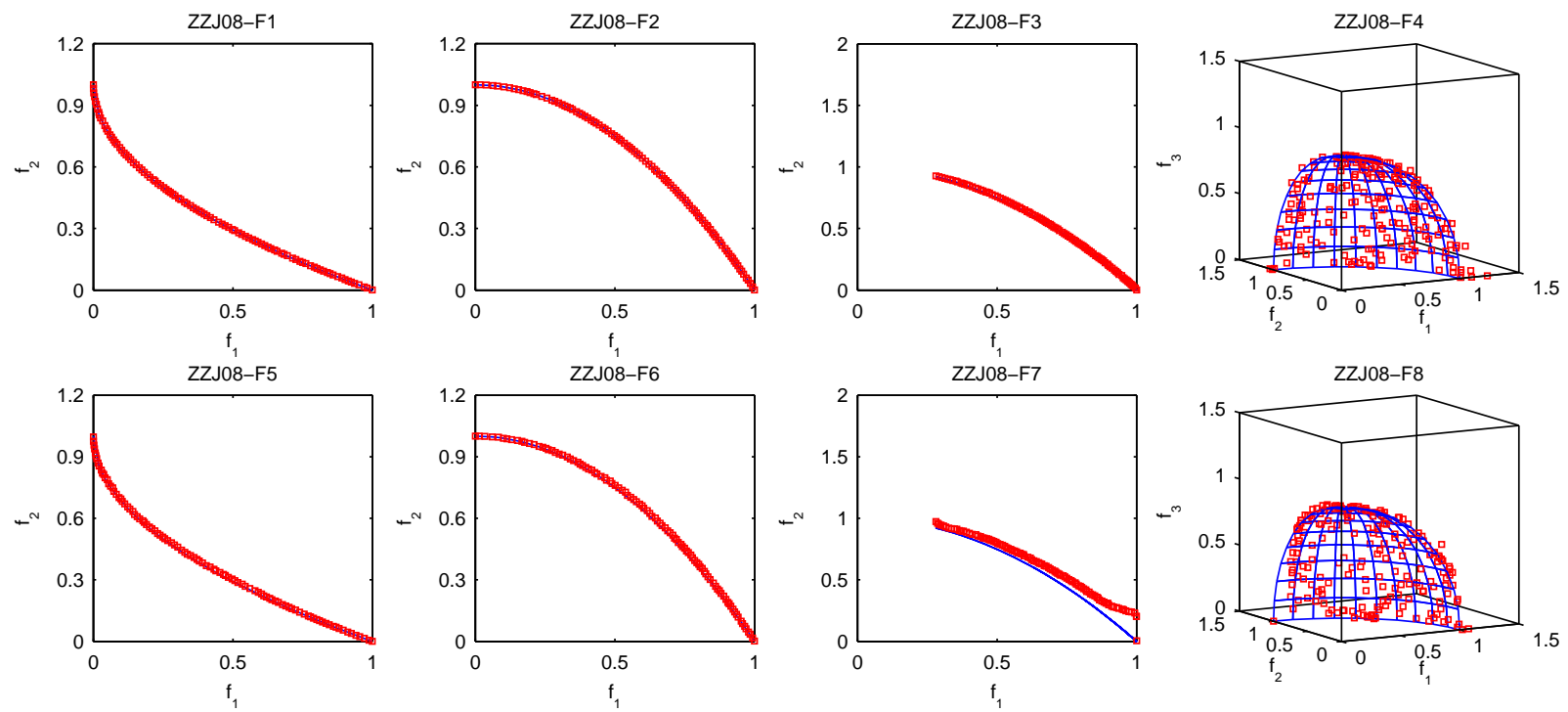

Fig. 12. The approximations with the lowest $I G D F$ values obtained by MMEA on regular MOPs.

\section{MORE Discussions}

\section{A. Can MMEA Solve Regular MOPs?}

MMEA was designed for solving an MOP of Class II, in which the dimensionality of its PS is not lower than the number of the objectives and unknown. In fact, MMEA uses the PCA technique to estimate the PS dimensionality before modeling the PS. Now a question arises whether MMEA can effectively solve a regular MOP in which the PS is an $(m-1)$ $\mathrm{D}$ continuous manifold in the decision space. To address this issue, we have compared MMEA with RM-MEDA on a set of regular MOP test instances with linear and nonlinear variable linkages introduced in [14], which are given in Table IV.

The experimental setting are the same as in [14]:

- the number of variables is $n=30$;

- the population size for each algorithm is $N=100$ for two objective instances, and 200 for three objective instances;

- the number of generations is 100 for ZZJ08-F1, ZZJ08F2, ZZJ08-F5 and ZZJ08-F6, 1,000 for ZZJ08-F3 and
ZZJ08-F7, and 200 for ZZJ08-F4 and ZZJ08-F8;

- the number of clusters in RM-MEDA $K=5$;

- $\alpha, \beta, \theta$ and $K_{\max }$ in MMEA are the same as in Table II.

All the following results are based on 20 independent runs of each algorithm on each test instance. In our experiments, 1,000 points, in which $x_{1}$ taking 1,000 equidistant values from their lower bounds to their upper bounds, are selected from the respective PFs of ZZJ08-F1 - ZZJ08-F3 and ZZJ08-F5 ZZJ08-F7 to form $P^{\star}$. $50 \times 50=2,500$ points in the PF of ZZJ08-F4 and ZZJ08-F8 with $x_{1}, x_{2}=\frac{0}{49}, \frac{1}{49}, \ldots, \frac{49}{49}$, are taken to form $P^{\star}$ for experiments on ZZJ08-F4 and ZZJ08F8. The bounded reference point is chosen to be $(1.5,1.5)^{T}$ for F1-F3 and F5-F7, and $(1.5,1.5,1.5)^{T}$ for F4 and F8 in calculating $I_{H}^{-}$values.

Table $\mathrm{V}$ gives the means and standard deviations of the $I_{H}^{-}$ and $I G D F$ values of the 20 final populations obtained by RMMEDA and MMEA for all the eight test instances. Figs. 11 and 12 show, in the objective space, the distribution of the final 
solutions obtained in the runs with the lowest $I G D F$ values of the two algorithms for these test instances, respectively.

It is clear from Table $\mathrm{V}$ that MMEA is significantly better than RM-MEDA on all the instances in terms of the $I_{H}^{-}$ metric, and MMEA significantly outperforms or is not worse than RM-MEDA in terms of $I G D F$ metric on these eight instances. The difference between the best approximations of RM-MEDA and those of MMEA in Figs. 11 and 12 can hardly be visually distinguished. These results imply that although MMEA is designed for Class II problems, its performance is not worse than RM-MEDA on regular MOPs.

\section{B. Can MMEA Deal with an MOP of Class I?}

An MOP of Class II has a continuous PS of dimensionality larger than $m-1$, while the PS of an MOP of Class I consists of a number of disconnected continuous manifolds. To investigate the ability of MMEA to tackle MOPs in Class I, we have tested MMEA on DT05-F4.4 [25], in which the two objectives to be minimized are as follows:

$$
\begin{aligned}
& f_{1}(x)=\sum_{i=1}^{n} \sin \left(\pi x_{i}\right), \\
& f_{2}(x)=\sum_{i=1}^{n} \cos \left(\pi x_{i}\right),
\end{aligned}
$$

and the search space is $[0,6]^{n}$. The PF of DT05-F4.4 is:

$$
f_{2}=-\sqrt{25-f_{1}^{2}}, f_{1} \in[-5,0],
$$

and its PS consists of $3^{n}$ disconnected parts, each of which is a line segment.

In our experiment on DT05-F4.4, $n$, the number of decision variables is set to be 5 , and $N$, the population size is 1,000 as in [25]. All the other parameter settings are the same as in Section IV. Fig. 13 presents the final population obtained in the run with the lowest $I G D F$ value among 20 independent runs. Clearly, MMEA has not produced a satisfactory approximation to the PS. This could be attributed to the fact that population clustering in MMEA is based on the distance in the objective space, which prevents it from distinguishing the different parts of the DT05-F4.4 PS in the decision space and thus cannot find a good approximation to its PS.

\section{Sensitivity of Control Parameters}

In the following, taking F3 as an example, we investigate the sensitivity of the four control parameters in MMEA.

1) The Effect of $\alpha$ : We have tried different values of $\alpha: 0.0$, $0.2,0.4,0.6,0.8,1.0,1.2,1.4,1.6,1.8$, and 2.0 for MMEA on F3. The settings of the other control parameters are the same as in Section IV. Fig. 14 shows the average $I G D F$ and $I G D X$ metrics v.s. the different values of $\alpha$, respectively. It is clear that MMEA works very well when $0.4 \leq \alpha \leq 2.0$. It also indicates that MMEA performs poorly if $\alpha$ is too small. This could be due to the fact that a small value of $\alpha$ might reduce the exploration ability of MMEA.

2) The Effect of $\beta$ : We have tried different values of $\beta$ : 0.0 , $0.2,0.4,0.6,0.8,1.0,1.2,1.4,1.6,1.8$, and 2.0 for MMEA on F3. The settings of the other control parameters are the same as in Section IV. Fig. 15 shows the average IGDF and IGDX metrics v.s. the different values of $\beta$, respectively. Clearly,
$I G D X$ value is more sensitive to $\beta$ than $I G D F$. This is not a surprise since $\beta$ is mainly for extending the search in the decision space. It is also evident that MMEA works well in terms of both $I G D X$ and $I G D F$ metrics if $0.8 \leq \beta \leq 2$.

3) The Effect of $\theta$ : We have tried different values of $\theta$ : $0.5,0.6,0.7,0.75,0.8,0.85,0.9,0.95$, and 1.0 on F3. The settings of the other parameters are the same as in Section IV. Fig. 16 shows the average $I G D F$ and $I G D X$ metrics v.s. the different values of $\theta$, respectively. It is clear from this figure that MMEA can approximate the PF of F3 if $0.5 \leq \theta \leq 0.95$ and it can approximate the PF of F3 if $0.75 \leq \theta \leq 0.95$. When $\theta=1.0$, the performance of MMEA becomes very poor. The reason might be that in such a case, MMEA implicitly assumes that the dimensionality of the PS is $n$ and set $\sigma^{k}$ to be zero in Step 5.6 in PCA and Modeling, as a result, the search ability of MMEA has been reduced significantly.

4) The Effect of $K_{\max }$ : In our experiment, we have tried the different values of $K_{\max }: 10,15,20,25,30,35,40$, 45, and 50. It is evident from Fig. 17 that MMEA could approximate the $\mathrm{PF}$ of $\mathrm{F} 3$ very well when $K_{\max } \geq 15$. However, when $K_{\max }>35$, the approximation quality in the decision space will decrease. It implies that with too many subpopulations, MMEA could not be able to correctly estimate the shape of the PS.

From the above experiments, we can also conclude that for each of these four control parameters, there is a reasonably large range such that MMEA works well.

\section{CPU Time Cost}

We have also recorded the CPU time used by each algorithm on F1-F7. The average CPU time ${ }^{3}$ used by the four algorithms are given in Table VI. Clearly, RM-MEDA needs much more CPU time than the three others. KP1 is the fastest in terms of CPU times. MMEA and Omni-Optimizer require about the same CPU time. The reason why MMEA is faster than RMMEDA might be that the PCA used in MMEA requires much less CPU time than the local PCA used in RM-MEDA.

\section{CONCLUSION}

A good approximation to both the PS and the PF of an MOP could be required in some real-world applications. A good approximation to the PF of an MOP might not represent a good approximation to the PS, for example, when the MOP in question is of Class I or II. Some effort has been made to approximate both the PS and the PF of an MOP of Class I. This paper represents a first attempt to do so for an MOP of Class II.

MMEA proposed in this paper generalizes the idea used in RM-MEDA to an MOP of Class II for approximating its PS and PF simultaneously. In the modeling phase of MMEA, the population is clustered into a number of subpopulations based on their distribution in the objective space, the PCA technique is then used to estimate the dimensionality of the PS manifold in each subpopulation, and then a probabilistic model

\footnotetext{
${ }^{3}$ The four algorithms are implemented in $\mathrm{C}++$, and they are executed in Thinkpad X60s with Intel Core Duo CPU L2400 1.66GHz, 2GB RAM, and Windows Vista.
} 

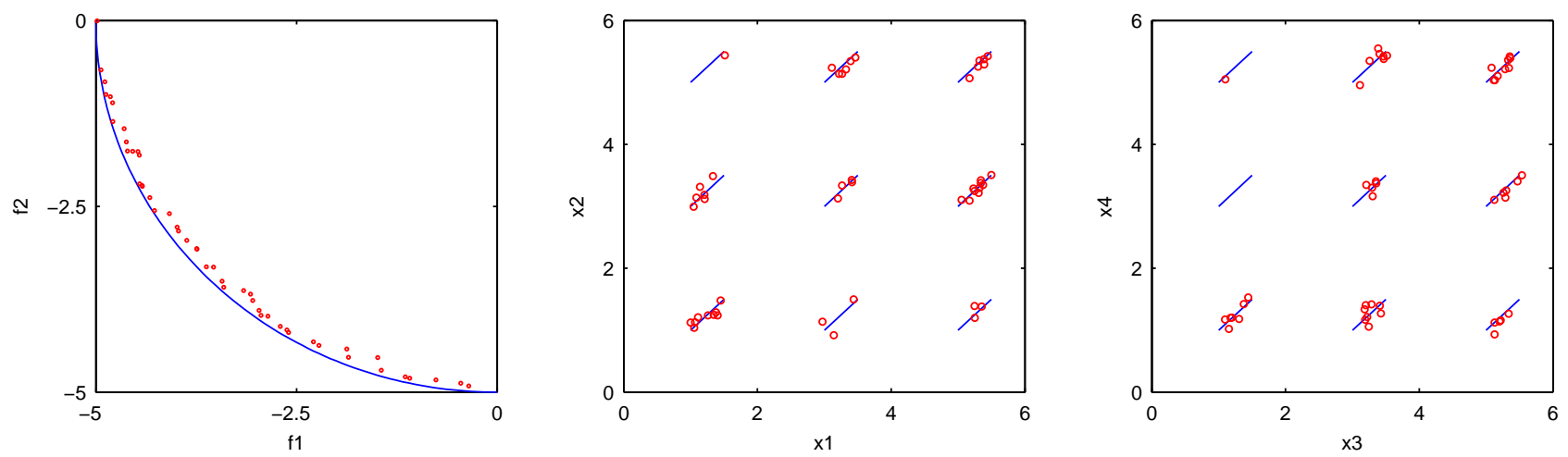

Fig. 13. The best approximation obtained in the 20 runs with the lowest $I G D F$ value by MMEA for DT05-F4.4. Left: the distribution of the final solutions in the objective space. Middle: the distribution of the final solutions in the $x_{1}-x_{2}$ space. Right: the distribution of the final solutions in the $x_{3}-x_{4}$ space.
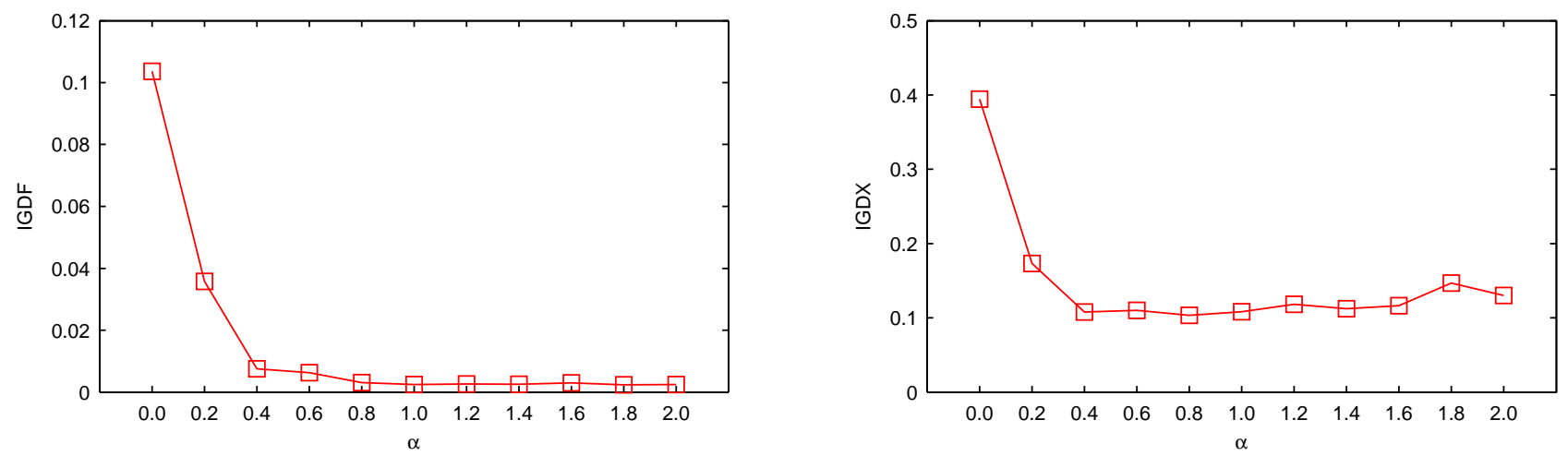

Fig. 14. The average IGD metrics of the 20 final approximations v.s. $\alpha$ in MMEA on F3.
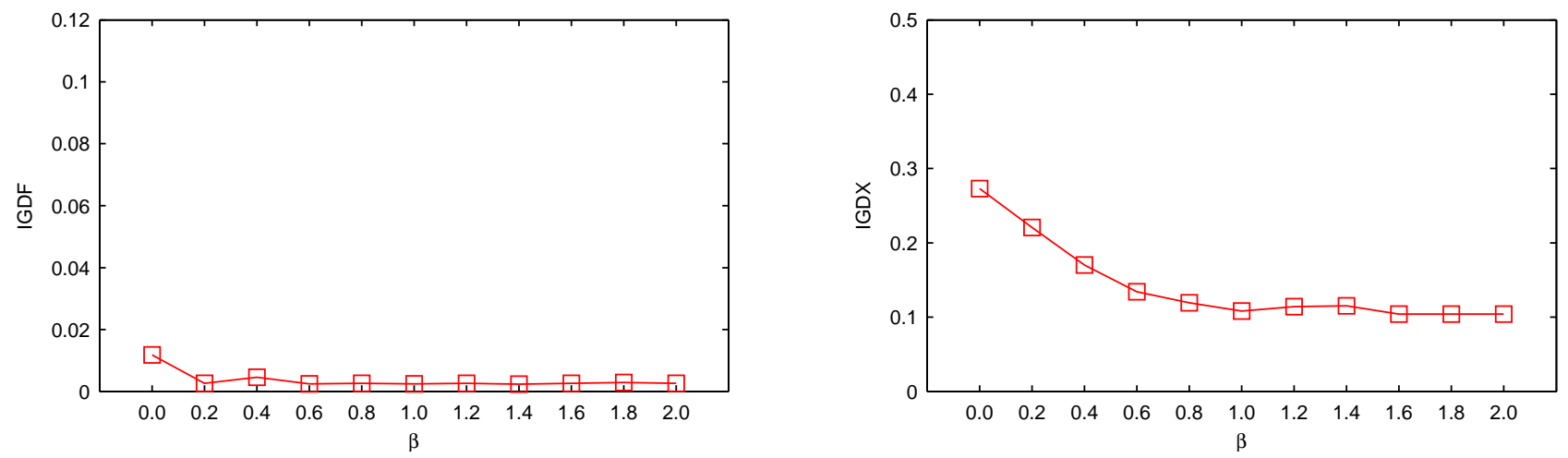

Fig. 15. The average IGD metrics of the 20 final approximations v.s. $\beta$ in MMEA on F3.
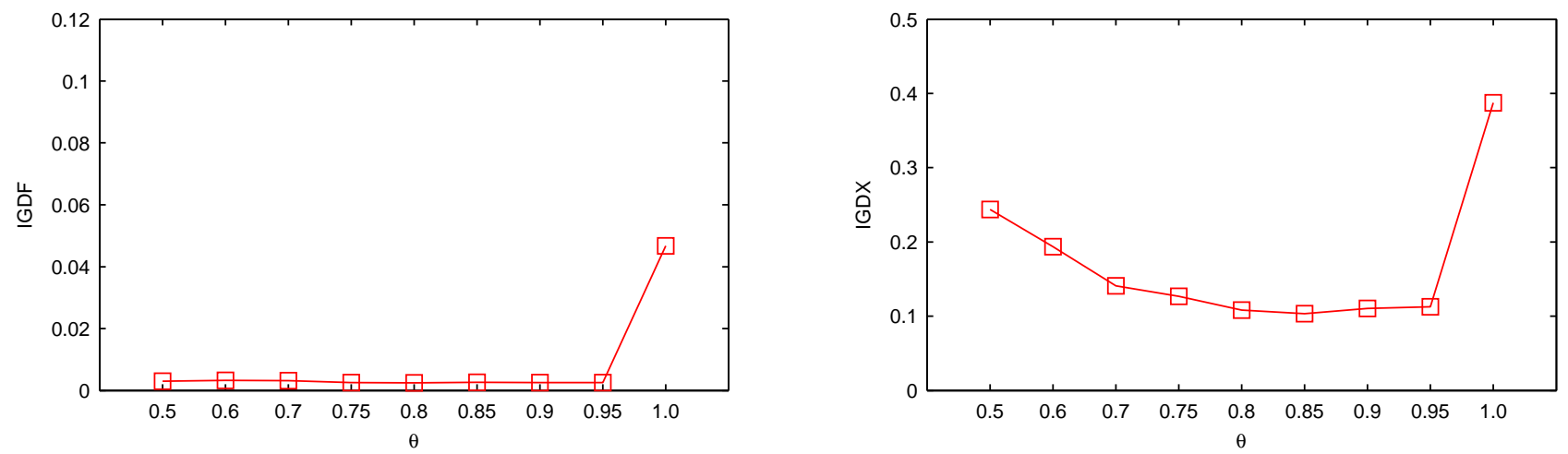

Fig. 16. The average IGD metrics of the 20 final approximations v.s. $\theta$ in MMEA on F3. 

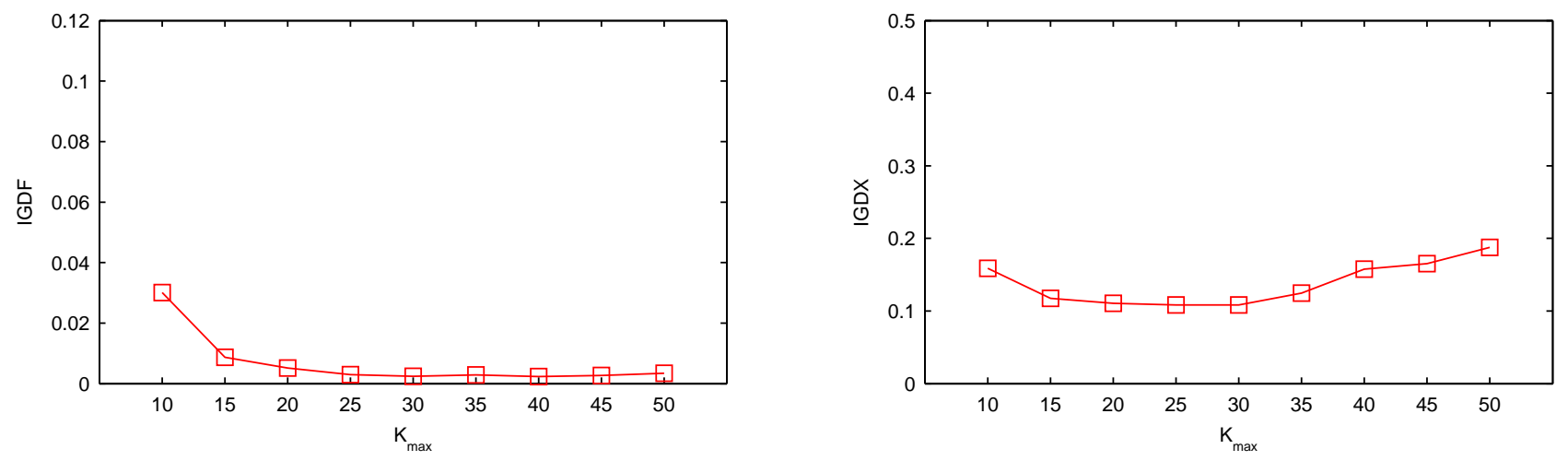

Fig. 17. The average IGD metrics of the 20 final approximations v.s. $K_{\max }$ in MMEA on F3.

TABLE VI

The Average CPU Time (IN SEConds) Used by Four Algorithms on F1-F7

\begin{tabular}{lrrrrrrr}
\hline \hline & F1 & F2 & F3 & F4 & F5 & F6 & F7 \\
\hline KP1 & 33.88 & 50.48 & 18.76 & 16.43 & 21.45 & 62.81 & 153.37 \\
& & & & & & & \\
Omni-Optimizer & 113.21 & 134.33 & 101.56 & 79.06 & 86.64 & 311.14 & 458.74 \\
& & & & & & & \\
RM-MEDA & 1339.91 & 1168.92 & 973.18 & 952.44 & 904.29 & 2743.44 & 2675.40 \\
& & & & & & & \\
MMEA & 136.18 & 165.22 & 128.66 & 125.10 & 120.80 & 413.86 & 470.12 \\
\hline \hline
\end{tabular}

is built for modeling the distribution of promising solutions in the decision space. We argue that such a modeling procedure could promote the population diversity in both the decision and objective spaces. New solutions are sampled from the model thus built. The population for the next generation is selected by the NDS-selection. The comparison between MMEA and the three other algorithms, KP1, Omni-Optimizer and RM-MEDA on seven test instances, five of which were proposed in this paper, has been made in this paper. Our empirical results have clearly indicated that MMEA has a big advantage over the three other algorithms in approximating both the PS and the $\mathrm{PF}$ of an MOP of Class II. We have investigated the ability of MMEA to deal with a regular MOP and an MOP of Class I. We have also studied the sensitivity of control parameters in MMEA.

The future research topics along this line may include:

- extension of MMEA to constrained MOPs, and MOPs under dynamic and/or noisy environment for approximating both their PS and PF [38]-[40].

- study of the scalability of MMEA to the numbers of decision variables and objectives [41]-[43].

- use of other machine learning methods in MMEA [44] [46].

- combination of other techniques, particularly, traditional mathematical programming methods and new ideas in MOEAs, with MMEA for improving the algorithm performance.

The C++ code of MMEA can be downloaded from Q. Zhang's homepage: http://dces.essex.ac.uk/staff/qzhang/.

\section{ACKNOWLEDGEMENT}

The authors thanks Dr. G. Eichfelder and the three anonymous reviewers for their very helpful and constructive comments.

\section{REFERENCES}

[1] K. Miettinen, Nonlinear Multiobjective Optimization, ser. Kluwer's International Series in Operations Research \& Management Science. Kluwer Academic Publishers, 1999.

[2] K. Deb, Multi-Objective Optimization using Evolutionary Algorithms. Baffins Lane, Chichester: John Wiley \& Sons, 2001.

[3] C. A. Coello Coello, D. A. van Veldhuizen, and G. B. Lamont, Evolutionary Algorithms for Solving Multi-Objective Problems. New York: Kluwer Academic Publishers, 2002.

[4] J. Knowles and D. Corne, "The Pareto archived evolution strategy: A new baseline algorithm for Pareto multiobjective optimisation," in Proceedings of the Congress on Evolutionary Computation, vol. 1. Washington D.C., USA: IEEE, 1999, pp. 98-105.

[5] M. Laumanns, L. Thiele, K. Deb, and E. Zitzler, "Combining convergence and diversity in evolutionary multiobjective optimization," Evolutionary Computation, vol. 10, no. 3, pp. 263-282, 2002.

[6] G. G. Yen and H. Lu, "Dynamic multiobjective evolutionary algorithm: Adaptive cell-based rank and density estimation," IEEE Transactions on Evolutionary Computation, vol. 7, no. 3, pp. 253-274, 2003.

[7] C. A. Coello Coello, G. T. Pulido, and M. S. Lechuga, "Handling multiple objectives with particle swarm optimization," IEEE Transactions on Evolutionary Computation, vol. 8, no. 3, pp. 256-279, June 2004.

[8] E. Zitzler and S. Künzli, "Indicator-based selection in multiobjective search," in Parallel Problem Solving from Nature - PPSN VIII, ser. Lecture Notes in Computer Science, vol. 3242. Birmingham, UK: Springer, September 2004, pp. 832-842.

[9] C. Gil, A. Márquez, R. Banos, M. G. Montoya, and J. Gómez, "A hybrid method for solving multi-objective global optimization problems," Journal of Global Optimization, vol. 38, no. 2, pp. 265-281, 2007.

[10] K. C. Tan, Y. J. Yang, and C. K. Goh, "A distributed cooperative coevolutionary algorithm for multiobjective optimization," IEEE Transactions on Evolutionary Computation, vol. 10, no. 5, pp. 527- 549, 2006. 
[11] C. Igel, N. Hansen, and S. Roth, "Covariance matrix adaptation for multi-objective optimization," Evolutionary Computation, vol. 15, no. 1, pp. 1-28, 2007.

[12] R. C. Purshouse and P. J. Fleming, "On the evolutionary optimization of many conflicting objectives," IEEE Transactions on Evolutionary Computation, vol. 11, no. 6, pp. 770-784, 2007.

[13] Q. Zhang and H. Li, "MOEA/D: A multiobjective evolutionary algorithm based on decomposition," IEEE Transactions on Evolutionary Computation, vol. 11, no. 6, pp. 712-731, 2007.

[14] Q. Zhang, A. Zhou, and Y. Jin, "RM-MEDA: A regularity model based multiobjective estimation of distribution algorithm," IEEE Transactions on Evolutionary Computation, vol. 12, no. 1, pp. 41-63, 2008.

[15] S. Bandyopadhyay, S. Saha, U. Maulik, and K. Deb, "A simulated annealing-based multiobjective optimization algorithm: AMOSA," IEEE Transactions on Evolutionary Computation, vol. 12, no. 3, pp. 269-283, 2008.

[16] K. I. Smith, R. M. Everson, J. E. Fieldsend, C. Murphy, and R. Misra, "Dominance-based multiobjective simulated annealing," IEEE Transactions on Evolutionary Computation, vol. 12, no. 3, pp. 323-342, 2008.

[17] H. P. Benson and S. Sayin, "Optimization over the efficient set: Four special cases," Journal of Optimization Theory and Applications, vol. 80, no. 1, pp. 3-18, 1994.

[18] P. T. Thach, H. Konno, and D. Yokota, "Dual approach to minimization on the set of Pareto-optimal solutions," Journal of Optimization Theory and Applications, vol. 88, no. 3, pp. 689-707, 1996.

[19] R. Horst and N. V. Thoai, "Utility function programs and optimization over the efficient set in multiple-objective decision making," Journal of Optimization Theory and Applications, vol. 92, no. 3, pp. 605-631, 1997.

[20] G. Eichfelder, Adaptive Scalarization Methods in Multiobjective Optimization. Springer, 2008.

[21] E. Zitzler, K. Deb, and L. Thiele, "Comparison of multiobjective evolutionary algorithms: Empirical results," Evolutionary Computation, vol. 8, no. 2, pp. 173-195, 2000

[22] Y. Jin, "Effectiveness of weighted sum of the objectives for evolutionary multi-objective optimization: Methods, analysis and applications," 2002, on-line available at: http://www.soft-computing.de/edwa2002.pdf.

[23] G. Rudolph, B. Naujoks, and M. Preuss, "Capabilities of EMOA to detect and preserve equivalent Pareto subsets," in Fouth International Conference on Evolutionary Multi-Criterion Optimization (EMO 2007), ser. Lecture Notes in Computer Science, vol. 4403. Matsushima, Japan: Springer, March 2007, pp. 36-50.

[24] S. Huband, P. Hingston, L. Barone, and L. While, "A review of multiobjective test problems and a scalable test problem toolkit," IEEE Transactions on Evolutionary Computation, vol. 10, no. 5, pp. 477-506, 2006.

[25] K. Deb and S. Tiwari, "Omni-optimizer: A procedure for single and multi-objective optimization," in Third International Conference on Evolutionary Multi-Criterion Optimization (EMO 2005), ser. Lecture Notes in Computer Science, C. A. Coello Coello, A. H. Aguirre, and E. Zitzler, Eds., vol. 3410. Guanajuato, Mexico: Springer, March 2005, pp. 41-65.

[26] K. Deb, A. Pratap, S. Agarwal, and T. Meyarivan, "A fast and elitist multiobjective genetic algorithm: NSGA-II," IEEE Transactions on Evolutionary Computation, vol. 6, no. 2, pp. 182-197, 2002.

[27] K. P. Chan and T. Ray, "An evolutionary algorithm to maintain diversity in the parametric and the objective space," in Third International Conference on Computational Intelligence, Robotics and Autonomous Systems(CIRAS 2005), Singapore, 2005.

[28] M. Preuss, B. Naujoks, and G. Rudolph, "Pareto Set and EMOA behavior for simple multimodal multiobjective functions," in Parallel Problem Solving from Nature - PPSN VIX, ser. Lecture Notes in Computer Science, vol. 4193. Reykjavik, Iceland: Springer, September 2006, pp. 513-522.

[29] L. Devroye, Non-Uniform Random Variate Generation. SpringerVerlag, 1986.

[30] N. Kambhatla and T. K. Leen, "Dimension reduction by local principal component analysis," Neural Computation, vol. 9, no. 7, pp. 1493-1516, October 1997.

[31] M. Reyes Sierra and C. A. Coello Coello, "A study of fitness inheritance and approximation techniques for multi-objective particle swarm optimization," in Proceedings of the Congress on Evolutionary Computation (CEC 2005). Edinburgh, U.K: IEEE Press, September 2005, pp. 65-72.

[32] J. D. Knowles, L. Thiele, and E. Zitzler, "A tutorial on the performance assessment of stochastic multiobjective optimizers," Computer Engineering and Networks Laboratory, ETH Zurich, Gloriastrasse 35, 8092 Zurich, Switzerland, Tech. Rep. 214, 2006.
[33] E. Zitzler and L. Thiele, "Multiobjective optimization using evolutionary algorithms - a comparative case study," in Parallel Problem Solving from Nature - PPSN V, ser. Lecture Notes in Computer Science, vol. 1498. Amsterdam, The Netherlands: Springer, September 1998, pp. 292-304.

[34] J. D. Knowles and D. W. Corne, "Properties of an adaptive archiving algorithm for storing nondominated vectors," IEEE Transactions on Evolutionary Computation, vol. 7, no. 2, pp. 100-116, 2003.

[35] E. Zitzler, M. Laumanns, and L. Thiele, "SPEA2: Improving the strength Pareto evolutionary algorithm for multiobjective optimization," in Evolutionary Methods for Design, Optimisation and Control. Barcelona, Spain: CIMNE, 2002, pp. 95-100.

[36] K. Deb and R. B. Agrawal, "Simulated binary crossover for continuous search space," Complex Systems, vol. 9, no. 2, pp. 115-148, 1995.

[37] K. Deb and M. Goyal, "A combined genetic adaptive search (GeneAS) for engineering design," Computer Science and Informatics, vol. 26, no. 4, pp. 30-45, 1996

[38] Y. Jin and J. Branke, "Evolutionary optimization in uncertain environments-A survey," IEEE Transactions on Evolutionary Computation, vol. 9, no. 3, pp. 303-317, 2005.

[39] A. Zhou, Y. Jin, Q. Zhang, B. Sendhoff, and E. Tsang, "Prediction-based population re-initialization for evolutionary dynamic multi-objective optimization," in Fouth International Conference on Evolutionary MultiCriterion Optimization (EMO 2007), ser. Lecture Notes in Computer Science, vol. 4403. Matsushima, Japan: Springer, March 2007, pp. 832-846.

[40] C. K. Goh and K. C. Tan, "An investigation on noisy environments in evolutionary multiobjective optimization," IEEE Transactions on Evolutionary Computation, vol. 11, no. 3, pp. 354-381, 2007.

[41] C. W. Ahn and R. S. Ramakrishna, "On the scalability of real-coded bayesian optimization algorithm," IEEE Transactions on Evolutionary Computation, vol. 12, no. 3, pp. 307-322, 2008.

[42] L. Martí, J. García, A. Berlanga, and J. M. Molina, "Scalable continuous multiobjective optimization with a neural network based estimation of distribution algorithm," in Applications of Evolutionary Computing: EvoWorkshops 2008, ser. Lecture Notes in Computer Science, M. Giacobini et al, Ed. Berlin/Heidelberg: Springer, 2008, no. 4974, pp. 535-544.

[43] Y. Jin, A. Zhou, Q. Zhang, B. Sendhoff, and E. Tsang, Multi-Objective Problem Solving. Springer, ch. Modeling Regularity to Improve Scalability of Model-based Multi-objective Optimization Algorithms, pp. 331-355.

[44] R. S. Michalski, "Learnable evolution model: Evolutionary processes guided by machine learning," Machine Learning, vol. 38, no. 1-2, pp. 9-40, 2000.

[45] R. Y. Rubinstein and D. P. Kroese, The Cross-Entropy Method: A Unified Approach to Monte Carlo Simulation, Randomized Optimization and Machine Learning. Springer Verlag, 2004.

[46] J. A. Lozano, P. Larranaga, I. Inza, and E. Bengoetxea, Eds., Towards a New Evolutionary Computation: Advances on Estimation of Distribution Algorithms. Secaucus, NJ, USA: Springer-Verlag New York, Inc., 2006. 


\title{
Point by Point Reply to the Reviewers
}

\author{
A. Zhou, Q. Zhang and Y. Jin
}

First of all, we would like to thank all the three reviewers for your very helpful and constructive comments.

Following your comments and suggestions, we have revised this paper.

\section{REPLY TO REVIEWER 1}

- "The manuscript describes solid, serious research. It has considerably improved. Most of my points of criticism have been addressed."

Reply: Thanks.

- "One main point of criticism remains. Still it is hard for me to judge the relevance of the "diverse pre-image"/"diverse Pareto set" problem. I repeat my comment from the previous review: Are there real-world applications reported in the literature where this problem has been identified as relevant by practitioners? I think just citing [17]-[19] and now [20] is not enough. I have dealt with several applications - but a diverse pre-image was never an issue."

Reply:

- This work was motivated by engineering design optimization. Suppose two objectives $f_{1}(x)$ and $f_{2}(x)$ are much more important than objective $v(x)$ where $x$ is decision (control) variables. One should first consider the following multiobjective optimization problem:

$$
\text { optimize }\left(f_{1}(x), f_{2}(x)\right)
$$

The decision maker often hopes to obtain a concrete mathematical description of the PS and PF (i.e., all the Pareto solutions) of the above problem for further analysis. But it is (nearly) impossible. Therefore, one should obtain a good approximation to the PS and the PF, and then optimize $v(x)$ in the PS subject to some other constraints.

- In micro heat exchanger optimization and blade optimization studied in Honda, $f_{1}$ and $f_{2}$ are pressure drop and heat transfer rate, and pressure loss and the variation of outlet pressure, respectively. The designers would like to have a good approximation to the PS so that they can choose solutions that are less costly, easier to manufacture and more robust to changes in the design as well as in environment.

- Approximation of the PS has been received attention from traditional math programming community and evolutionary computation.

- We have revised the second paragraph in $\mathrm{RC}$ in page 1 to explain our motivation.

- "When I asked the authors to comment on "If diversity in the decision space is a goal not reflected in the objectives, then the problem is casted wrongly", I obviously did not make my point clear. If diversity in the pre-image is an issue, then one might argue that this demand should be expressed by an additional objective."

Reply: We think that it is very hard to express this demand by an additional objective for the following reasons:

- Consider

$$
\text { minimize } F(x)=\left(f_{1}(x), f_{2}(x)\right)
$$

One might consider two computational tasks for the above MOP:

1) finding a set of solutions to approximate its PF as most popular MOEAs do.

2) finding a set of solutions to approximate both the PS and PF as some researchers including us have attempted to do.

Suppose the size of the set is fixed to be 100. Let the set of solutions be:

$$
S=\left\{x^{1}, \ldots, x^{100}\right\}
$$

where each $x^{i}$ is a point in the decision space. Suppose that we use div to measure the diversity of $S$, or how good $S$ approximates the PS. Therefore, one of our goals in task (2) is to optimize $\operatorname{div}(S)$.

- Since $\operatorname{div}(S)$ is the function of $S$, but not decision variable $x$, therefore, it is hard for us to consider $\operatorname{div}(S)$ and $f_{1}(x), f_{2}(x)$ at the same level. In other words, it is hard to set $\operatorname{div}(S)$ as the third objective.

- "Minor comments:

Footnote page 1: What does "relatively uniformly distributed" (mathematically) mean?"

Reply: We deleted it and reworded Paragraph 2 in LC in page 1.

- "I still think that "hyper-cuboid" is a better word, because it makes clear that the interior is considered." 
Reply: Corrected.

- "When referring to particular statements in a book, the corresponding pages or chapters should be given in the citation instead of just citing the book."

Reply: We used the IEEE latex style file. We have put the page number in the bib file, but it seems that IEEEstyle file doesn't support it.

- “Typos: Ref. [11] "evolutioanry" - > "evolutionary" Ref. [23][38] ",Japan" - > ", Japan”"

Reply: Corrected.

\section{REPLY TO REVIEWER 2}

- "The manuscript has been well revised on the basis of reviewer's comments. Still, there are more comments that improve its quality."

Reply: Thanks.

- “- MMEA has mainly targeted on the problems of Class II, and thus further concrete descriptions on the Class II must be presented in Section I."

Reply: Bullet point 2 in $\mathrm{RC}$ in page 1 has been revised.

- "- In computational results, it is desirable to clearly state which test problem belongs to which class."

Reply: In the caption of Table I, we have stated that all the test instances in this table belong to Class II.

- “- Why does MMEA well approximate PF as well as PS? In my understanding, clustering in the objective space and using PCA for model building are key factors. It seems that such procedures transform a class-II problem into a class-I problem within each cluster. If this investigation is not ture, please offer proper, intuitive reasons; but it does not mean rigorous mathematical support."

Reply: We have added a paragraph before bullet points in Page 4 to explain why our method can approximate the PS and the PF.

- “- From Tables III and V, it is hard to say that MMEA significantly outperforms RM-MEDA. As such, my previous assertion on statistical test on performances is still valid."

Reply: The one side $t$-test has been made on results in table III and V in this version.

- “- In Section V. C, the effect of control parameters has been empirically investigated with F3. Do the results hold up for other test problems such as F2, F5, and so forth?"

Reply: We have done experiments on F2 and F5. The results are similar. Due to the page limit, we decide not to include these results in the paper. The figures attached to this reply show our results on F2 and F5.

\section{REPLY TO REVIEWER 3}

- "The revised version of the paper complies with most of my concerns of the first version. I have some minor comments:" Reply: Thanks.

- "page 1, column 2: Class I "...the same dimensionality. Therefore the PS could consist of a number of disconnected continuous manifolds"

"I do not think that what you say in the second sentence can be deduced from what you say in the first sentence".

Reply: It has been deleted.

- page 2, column 1: "Chan and Ray suggested using two selection operators in MOEAs, one encourages the diversity in the objective space and the other does so in the decision space [27]."

[27] should be after Chan and Ray

Reply: corrected.

- *page2, column 2: Phase 3 modeling

modeling should be "Modeling"

Reply: corrected.

- *page3, column 1: "...We require that reference points uniformly spread on the Utopian PF..."

I think this sentence should be written

Reply: It has been rewritten.

- "*page3, column 1: "..Utopian PF is a simplex, in our implementation, we use the simplex point picking method ${ }^{2} . . "$ $I$ would prefer a reference of a book more than a reference in wiki

Reply: corrected.

- * page4, column 1: Step 5.5 at the end of the formula there is an extra ","

Reply: corrected.

- * page4, column 2: "...The crowding distance, used in Step 3, of point $x$ in $S$ is defined as the average side length of the largest $m-D$ rectangle in the objective space subject to two constraints: (a) each of its sides is parallel to a coordinate axis, and..”

What is $S$ ? 
Reply Sorry for the typo. It has been corrected.

- * page18, column2: "Can MMEA Deal..." D should be in lower case

Reply: It seems to us that in IEEE Trans papers, The first letter in a subsubsection title should be in capital.

- * page 19, column2: "...MMEA is faster than RM-MEDA is mainly because that the PCA used..." The sentence should be corrected

Reply: It has been corrected. 

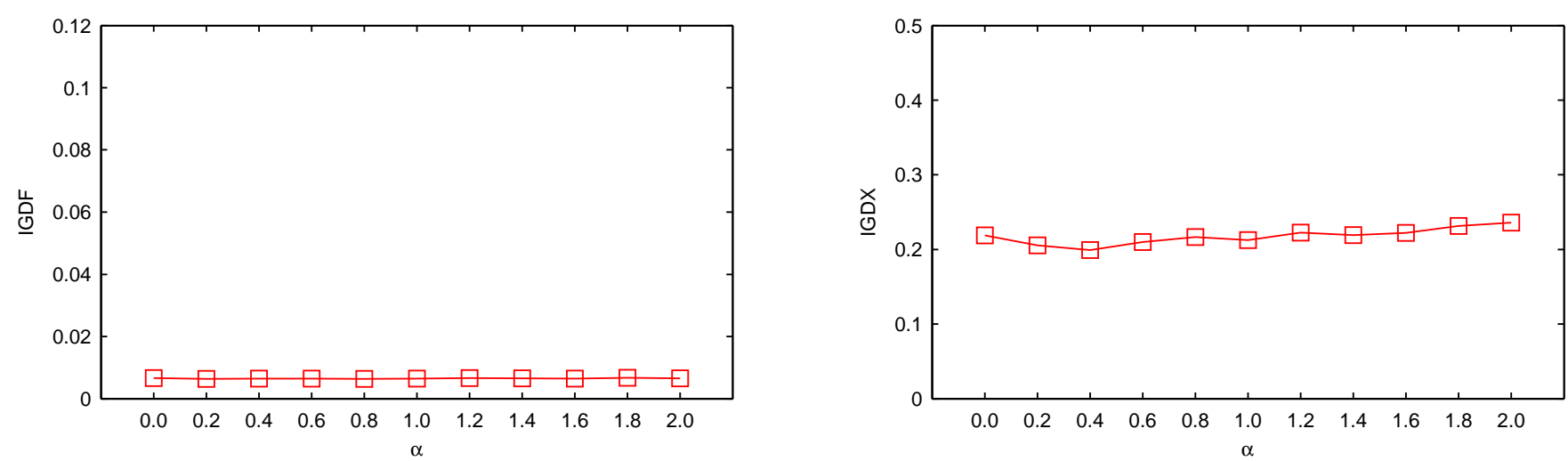

(a) The average IGD metrics of the 20 final approximations v.s. $\alpha$ in MMEA on F2.
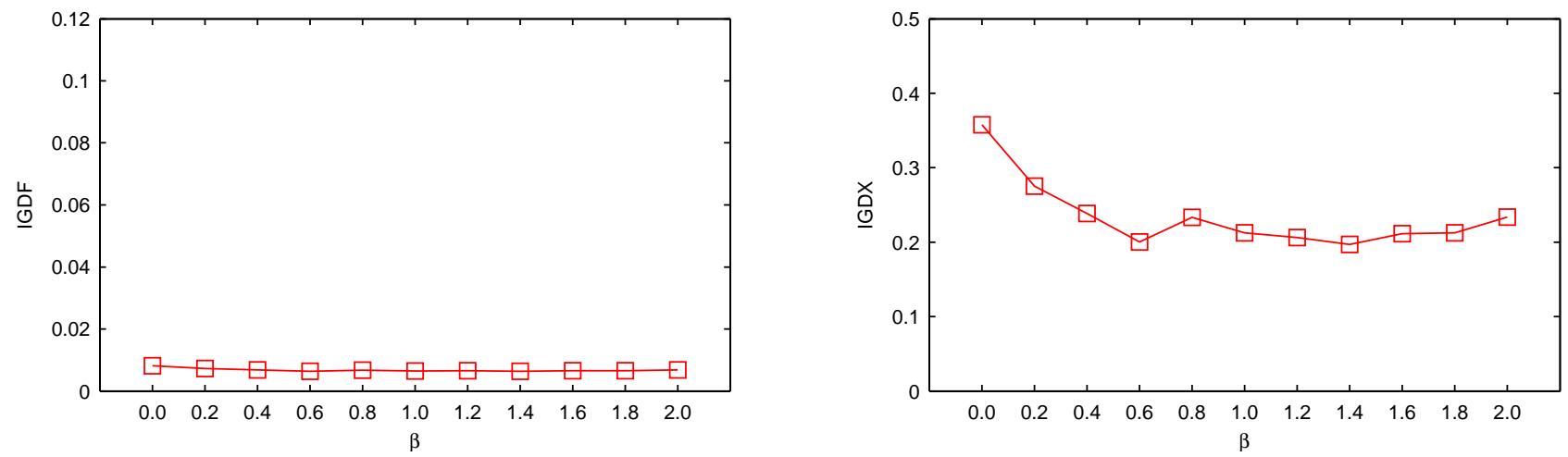

(b) The average IGD metrics of the 20 final approximations v.s. $\beta$ in MMEA on F2.
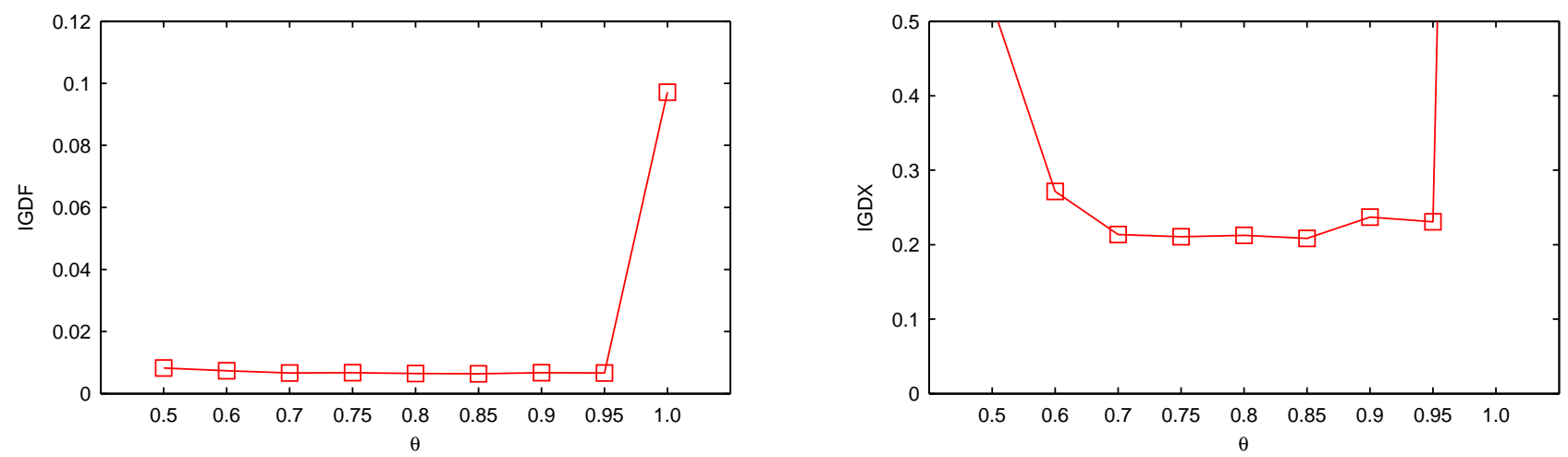

(c) The average IGD metrics of the 20 final approximations v.s. $\theta$ in MMEA on F2.
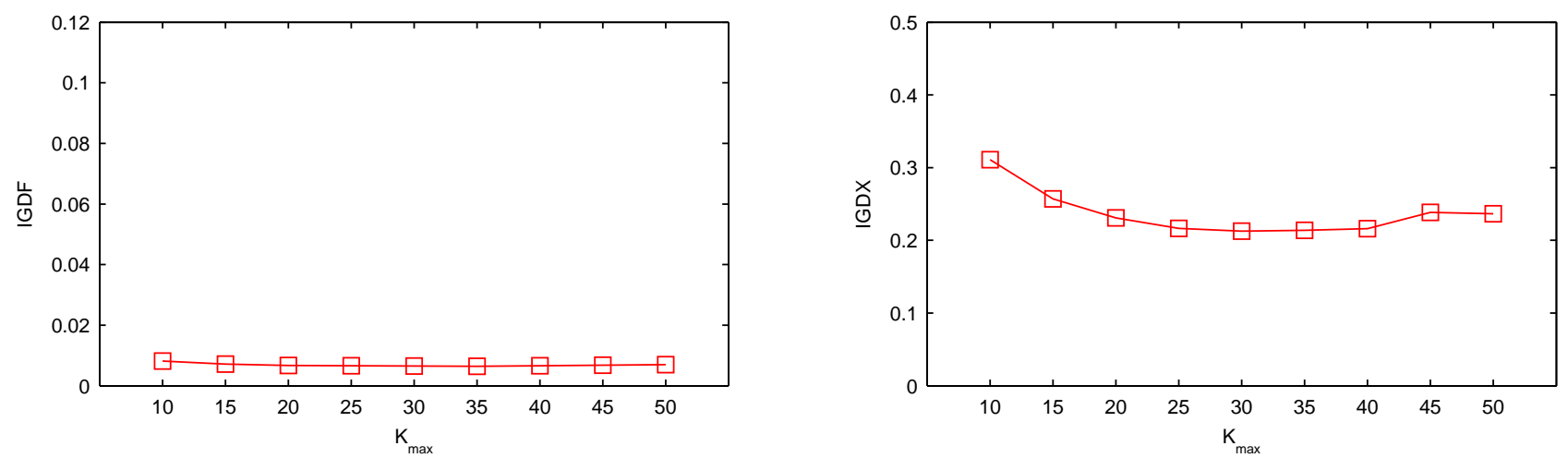

(d) The average IGD metrics of the 20 final approximations v.s. $K_{\max }$ in MMEA on F2.

Fig. 1. Sensitivity of Control Parameters on F2. 

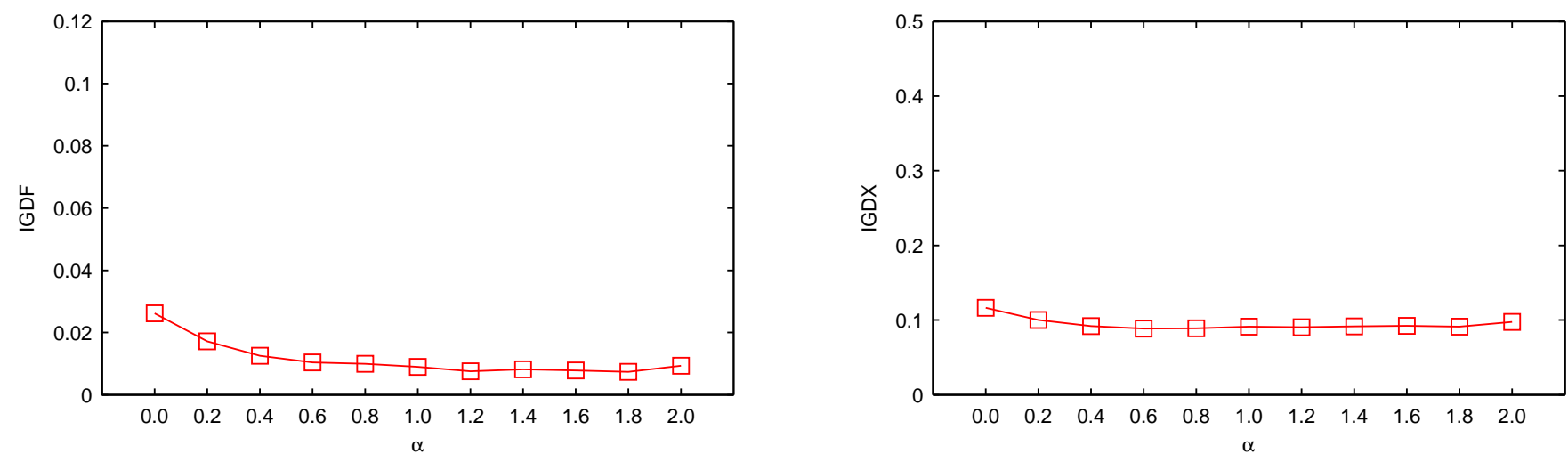

(a) The average IGD metrics of the 20 final approximations v.s. $\alpha$ in MMEA on F5.
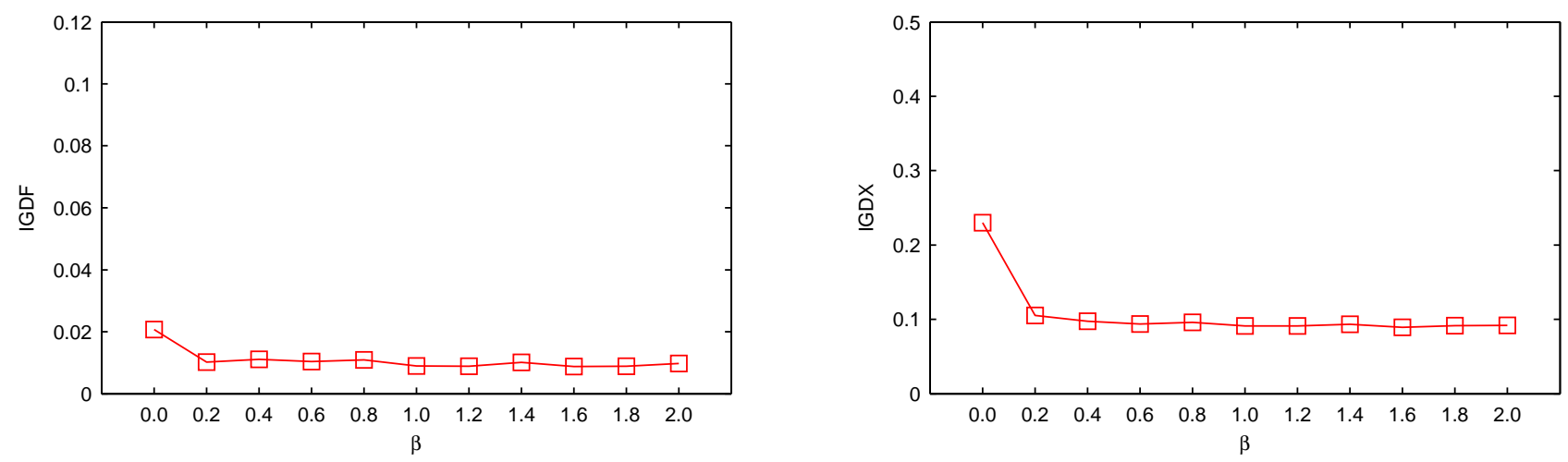

(b) The average IGD metrics of the 20 final approximations v.s. $\beta$ in MMEA on F5.
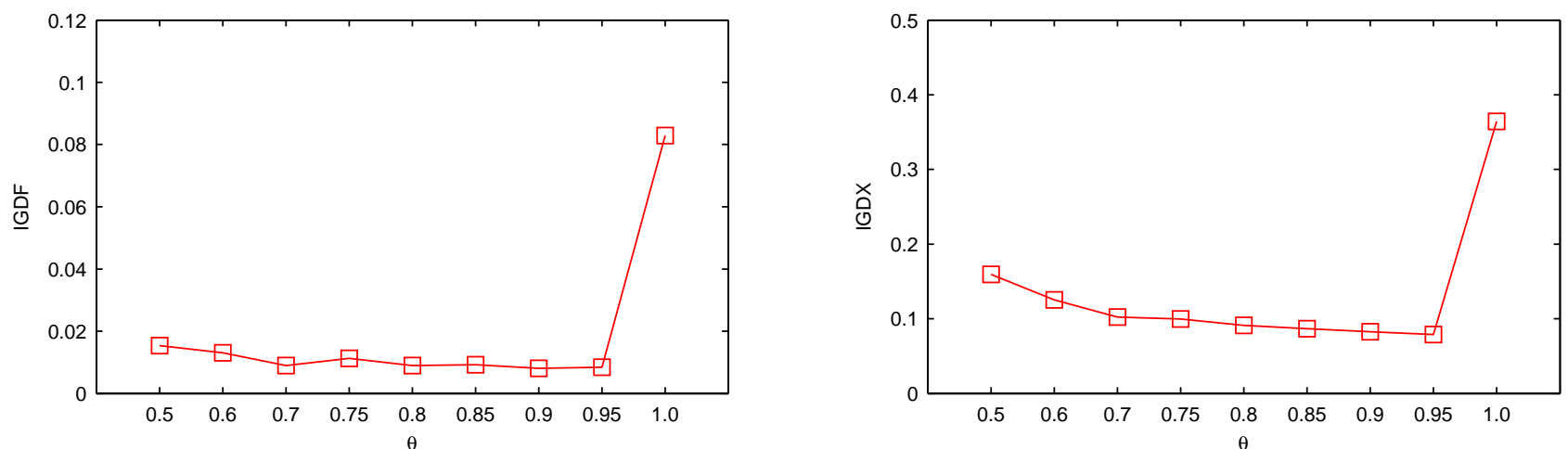

(c) The average IGD metrics of the 20 final approximations v.s. $\theta$ in MMEA on F5.
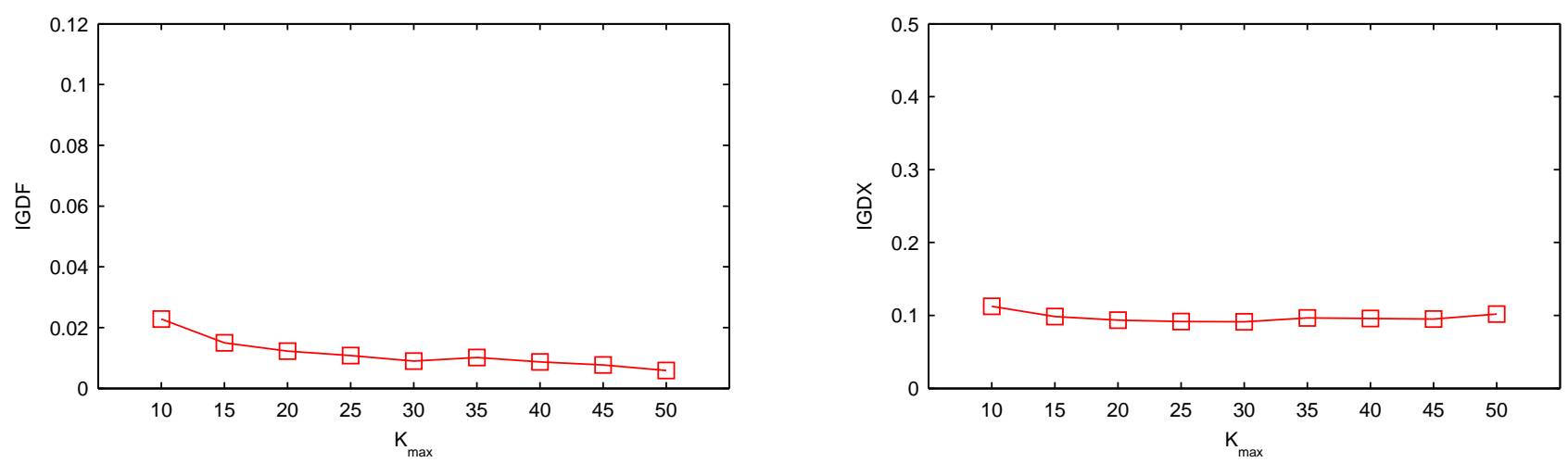

(d) The average IGD metrics of the 20 final approximations v.s. $K_{\max }$ in MMEA on F5.

Fig. 2. Sensitivity of Control Parameters on F5. 